\title{
NEWTON AND CONJUGATE GRADIENT FOR HARMONIC MAPS FROM THE DISC INTO THE SPHERE
}

\author{
Morgan PiERre ${ }^{1}$
}

\begin{abstract}
We compute numerically the minimizers of the Dirichlet energy

$$
E(u)=\frac{1}{2} \int_{B^{2}}|\nabla u|^{2} \mathrm{~d} x
$$

among maps $u: B^{2} \rightarrow S^{2}$ from the unit disc into the unit sphere that satisfy a boundary condition and a degree condition. We use a Sobolev gradient algorithm for the minimization and we prove that its continuous version preserves the degree. For the discretization of the problem we use continuous $P_{1}$ finite elements. We propose an original mesh-refining strategy needed to preserve the degree with the discrete version of the algorithm (which is a preconditioned projected gradient). In order to improve the convergence, we generalize to manifolds the classical Newton and conjugate gradient algorithms. We give a proof of the quadratic convergence of the Newton algorithm for manifolds in a general setting.
\end{abstract}

Mathematics Subject Classification. 58E20, 78M10, 65N30, $90 \mathrm{C} 53$.

Received January 28, 2003.

\section{INTRODUCTION}

This paper is devoted to the numerical computation of minimizing harmonic maps from the unit disc $B^{2}$ into the unit sphere $S^{2}$ with boundary condition and degree condition.

More precisely, we define the Sobolev space [15]

$$
H_{g}^{1}\left(B^{2}, S^{2}\right):=\left\{u \in H^{1}\left(B^{2}, \mathbb{R}^{3}\right),|u|=1 \text { a.e., } u_{\mid \partial B^{2}}=g \text { in the sense of trace }\right\},
$$

where $B^{2}=\left\{(x, y) \in \mathbb{R}^{2}, x^{2}+y^{2}<1\right\}$ is the unit disc, $S^{2}=\left\{u \in \mathbb{R}^{3},|u|=1\right\}$ is the unit sphere and the boundary condition $g$ belongs to $\mathcal{C}^{1}\left(\partial B^{2}, S^{2}\right)$. We want to minimize numerically the Dirichlet energy

$$
E(u)=\frac{1}{2} \int_{B^{2}}|\nabla u|^{2} \mathrm{~d} x \mathrm{~d} y
$$

for $u$ in connected components of $H_{g}^{1}\left(B^{2}, S^{2}\right)$, in order to find (approximations of) harmonic maps.

Keywords and phrases. Harmonic maps, finite elements, mesh-refinement, Sobolev gradient, Newton algorithm, conjugate gradient.

1 Centre de Mathématiques et de Leurs Applications, École Normale Supérieure de Cachan, 61 avenue du Président Wilson, 94235 Cachan Cedex, France; e-mail: Morgan.Pierre@cmla.ens-cachan.fr 
Recall that a map $u \in H_{g}^{1}\left(B^{2}, S^{2}\right)$ is harmonic if it is a critical point of $E$ or equivalently if it satisfies the following equation in the sense of distributions:

$$
-\Delta u=u|\nabla u|^{2} \text { in } \mathcal{D}^{\prime}(\Omega)
$$

The theory of harmonic maps has been considered by numerous authors (see the report [12]) and is related to simplified models in the theory of liquid crystals [1,8]. However, numerical computations are difficult because of the condition $|u(x)|=1$ and the only efficient algorithm is a preconditionned projected gradient given by Alouges [1].

Let us first recall the theoretical results on the problem. Following Brézis and Coron [5] we define the connected components of $H_{g}^{1}\left(B^{2}, S^{2}\right)$ as homotopy classes. Let

$$
Q(u)=\frac{1}{4 \pi} \int_{B^{2}} u \cdot\left(u_{x} \wedge u_{y}\right) \mathrm{d} x \mathrm{~d} y
$$

and choose a reference map $\underline{u} \in H_{g}^{1}\left(B^{2}, S^{2}\right)$. For $u \in H_{g}^{1}\left(B^{2}, S^{2}\right), Q(u)-Q(\underline{u})$ is an integer called the degree of the map $u$ with respect to $\underline{u}$. It is actually the degree of the map

$$
u \star \underline{u}(z):= \begin{cases}u(z) & \text { if }|z| \leq 1 \\ \underline{u}(1 / \bar{z}) & \text { if }|z| \geq 1\end{cases}
$$

from $S^{2} \simeq \mathbb{C}$ into $S^{2}$ which is obtained by "gluing together" $u$ and $\underline{u}$. The homotopy classes

$$
\mathcal{E}_{g, p}=\left\{u \in H_{g}^{1}\left(B^{2}, S^{2}\right), Q(u)-Q(\underline{u})=p\right\}
$$

for $p \in \mathbb{Z}$ are the connected components of $H_{g}^{1}\left(B^{2}, S^{2}\right)$.

The space $H_{g}^{1}\left(B^{2}, S^{2}\right)$ is not empty and it is easy to see (by using a minimizing sequence) that there exists an absolute minimizer for $E$ in $H_{g}^{1}\left(B^{2}, S^{2}\right)$ (not necessarily unique) which is a harmonic map. In the following we use an absolute minimizer as our reference map $\underline{u}$ for the numbering of the degree: with this convention the class $\mathcal{E}_{g, 0}$ always has a minimizer. The hope is to obtain other harmonic maps by minimizing $E$ in $\mathcal{E}_{g, p}$. However, depending on the boundary condition and the degree, this problem may have a solution or not.

When $g$ is constant, Lemaire [19] proved that the only harmonic extension of $g$ is the constant map. When $g$ is not constant, Brézis and Coron [5] and Jost [16] showed that at least one of the classes $\mathcal{E}_{g, 1}$ or $\mathcal{E}_{g,-1}$ admits a minimizer.

A few years later, Soyeur [30] gives a generalization of this result by using the holomorphic structure of $S^{2}: B^{2}$ is considered as a subset of $\mathbb{C}$ and $S^{2}$ is identified to $\mathbb{C}$ via the stereographic projection of south pole. The key example is the following: if $g(z)=a z^{n}$ with $a>0$ small enough, then $u^{+}(z)=a z^{n}$ is the absolute minimizer (with degree 0 ), $u^{-}(z)=\frac{a}{\bar{z}^{n}}$ is the minimizer in $\mathcal{E}_{g,-n}$ and the class $\mathcal{E}_{g, p}$ admits a minimizer if and only if $p \in[-n, 0]$.

Qing [25] and Kuwert [18] completed the result of Soyeur and independently established the following theorem, where we use the notation

$$
\Lambda_{p}:=\inf _{u \in \mathcal{E}_{g, p}} E(u)
$$

Theorem 1.1. Let $u^{+}\left(u^{-}\right.$, respectively) be the meromorphic (anti-meromorphic) extension of $g$ from $\partial B^{2}$ to $B^{2}$, and let $p^{+} \geq 0$ be the degree of $u^{+}\left(p^{-} \leq 0\right.$ the degree of $\left.u^{-}\right)$; we set $p^{+}=+\infty\left(p^{-}=-\infty\right)$ if the extension $u^{+}\left(u^{-}\right)$does not exist.

Then $\Lambda_{p}$ is achieved if and only if $p \in\left[p^{-}, p^{+}\right]$. Moreover, let $\left(u^{k}\right)_{k}$ be a minimizing sequence in $\mathcal{E}_{g, p}$ relative to $\Lambda_{p}$ that converges weakly in $H^{1}$ to some $u \in H_{g}^{1}\left(B^{2}, S^{2}\right)$. 
For $p \geq p^{+}$we have $u=u^{+}$and $\Lambda_{p}=\Lambda_{p^{+}}+4 \pi\left|p-p^{+}\right|$(for $p \leq p^{-}$respectively, we have $u=u^{-}$and $\left.\Lambda_{p}=\Lambda_{p^{-}}+4 \pi\left|p-p^{-}\right|\right)$.

For $p \in\left[p^{-}, p^{+}\right]$the sequence $\left(u^{k}\right)_{k}$ converges strongly to $u$ which minimizes the energy in $\mathcal{E}_{g, p}$.

In order to conclude these theoretical results, let us point out that any minimizer of $E$ in $\mathcal{E}_{g, p}$ is smooth up to the boundary $[22,27]$.

What happens when $p>p^{+}$? The heuristic reason for which a minimizing sequence in $\mathcal{E}_{g, p}$ with $p>p^{+}$ does not converge strongly is that it concentrates the energy on a finite number of points in $\overline{B^{2}}$; on each of these points the "limit map" covers the sphere a finite number of times, which explains how the degree is lost and why the gap in the energy is a multiple of $4 \pi$. In order to describe this concentration phenomenon it is necessary to enlarge the class of admissible solutions and to consider the convergence in the sense of graphs: this is the quite technical concept of cartesian currents introduced by Giaquinta, Modica and Souček [10,11].

In the following, we compute numerically the regular minimizers which are not known explicitely for the boundary condition $g(z)=a z^{n}$.

We first use the very general algorithm [1] to obtain minimizing sequences and we prove that it preserves the degree in a continuous setting. We use continuous $P_{1}$ elements to discretize the problem. The discretization which is based on a triangulation of the disc gives good results for the computation of the absolute minimiser. However for the other regular minimizers we need a mesh-refinement method in order to guarantee the preservation of the degree. Indeed, because of the $P_{1}$ elements, the constraint $\left|u^{h}(x)\right|=1$ is respected for the discretized solution $u^{h}$ only at the nodes of the triangulation. The mesh-refinement method we use is the usual "cut the triangles in 4" method, but the error estimator we use is original and geometric: we impose a maximum edge length in the image mesh living in $S^{2}$. We observe the limits of the refinement-method in the computation of singular solutions.

Since the original algorithm [1] requires a great number of iterations in the case of a solution with strong gradients, we improve its speed of convergence. By interpreting this algorithm as a Sobolev gradient [23] with step-size 1 on a Riemannian submanifold, we develop a Newton algorithm and conjugate gradient algorithm [20] for Riemannian manifolds. In the appendix, we give a proof of the quadratic convergence of the Newton algorithm for manifolds in a general setting.

\section{SobOLEV GRADIENT FOR HARMONIC MAPS}

In this section we first recall the algorithm for finding minimizing harmonic maps [1]. We interpret this algorithm in the continuous case as a (projected) Sobolev gradient [23] and show that it preserves the degree. We then give the discretization of the algorithm by $P_{1}$ finite element discretization: in numerical interpretation, the algorithm is a (projected) gradient computed with a preconditionned conjugate gradient method. We explain how to implement the discretized algorithm in our $2 \mathrm{~d}$ problem by adding a refinement technique in order to preserve the degree. We finally comment the numerical results.

\subsection{The continuous algorithm}

The key point of the algorithm is to define for every $u \in H_{g}^{1}\left(B^{2}, S^{2}\right)$ a "tangent space":

$$
T_{u}:=\left\{w \in H_{0}^{1}\left(D^{2}, \mathbb{R}^{3}\right) \text { such that } w(x) \cdot u(x)=0 \text { a.e. }\right\}
$$

Notice that $T_{u}$ is a closed subspace of $H_{0}^{1}\left(B^{2}, \mathbb{R}^{3}\right)$ and thus it is a Hilbert space for the usual scalar product on $H^{1}\left(D^{2}, \mathbb{R}^{3}\right)$. Let $a(\cdot, \cdot)$ be the continuous symmetric bilinear form on $H^{1}\left(D^{2}, \mathbb{R}^{3}\right)$ :

$$
a(u, v)=\int_{B^{2}} \nabla u \cdot \nabla v \mathrm{~d} x \mathrm{~d} y \quad \forall u, v \in H^{1}\left(B^{2}, \mathbb{R}^{3}\right) .
$$


By the Poincaré inequality $a(\cdot, \cdot)$ is coercive on $H_{0}^{1}\left(B^{2}, \mathbb{R}^{3}\right)$ (it is actually the natural scalar product on $H_{0}^{1}$ ) and $a$ fortiori on $T_{u}$. By the Lax-Milgram theorem (see [4]), the problem:

$$
\text { Find } w \in T_{u} \text { such that } a(w, \varphi)=a(u, \varphi) \quad \forall \varphi \in T_{u}
$$

has a unique solution which is also the unique solution to the problem:

$$
\text { Minimize } E(u-w) \text { for } w \in T_{u} \text {. }
$$

The algorithm [1] reads as follows:

Algorithm 2.1 (Projected Sobolev gradient).

Initial Data. Set $k=0$ and choose $u^{k} \in H_{g}^{1}\left(B^{2}, S^{2}\right)$ with given degree.

Step 1. Find $w^{k} \in T_{u^{k}}$ such that

$$
a\left(w^{k}, \varphi\right)=a\left(u^{k}, \varphi\right) \quad \forall \varphi \in T_{u^{k}}
$$

Step 2. Set

Replace $k$ by $k+1$ and go to step 1 .

$$
u^{k+1}=\frac{u^{k}-w^{k}}{\left|u^{k}-w^{k}\right|}
$$

\subsubsection{Projected Sobolev gradient}

Let $u \in H_{g}^{1}\left(B^{2}, S^{2}\right)$ and $\varphi \in T_{u}$. Then $E$ being a continuous quadratic form on $H^{1}\left(B^{2}, \mathbb{R}^{3}\right)$ is differentiable and

$$
\left.\frac{\mathrm{d} E}{\mathrm{~d} t}(u+t \varphi)\right|_{t=0}=a(u, \varphi)
$$

This gives an interpretation of the solution $w^{k}$ of step 1 as the gradient of $E$ in the tangent space $T_{u^{k}}$ with respect to the scalar product on $H_{0}^{1}$ (given by $a(\cdot, \cdot)$ ). The idea here is to choose the $H_{0}^{1}$ scalar product instead of the $L^{2}$ scalar product which is usual in distributions: such a gradient $w^{k}$ is called Sobolev gradient. The numerical computation of this gradient which we will see in the next section, is made by a preconditionned conjugate gradient (the preconditionning being given by the scalar product $H_{0}^{1}$ ).

Since we project the result on the sphere in step 2, the Algorithm 2.1 is then a projected Sobolev gradient algorithm (see [2]) with step-size 1 and this step-size is almost optimal because of the Sobolev gradient.

We recall the important convergence result of this algorithm:

Theorem 2.1 (Alouges [1]). Step 1 and step 2 are energy decreasing and the algorithm converges in the sense that (up to a subsequence) $\left(u^{k}\right)$ weakly converges in $H^{1}\left(B^{2}, \mathbb{R}^{3}\right)$ to a harmonic map $u \in H_{g}^{1}\left(B^{2}, S^{2}\right)$.

\subsubsection{Preservation of the degree}

It is important in our problem to know that $u^{k+1}$ and $u^{k}$ have the same degree. The following proposition implies that the map $[0,1] \ni t \rightarrow \frac{u^{k}+t w^{k}}{\left|u^{k}+t w^{k}\right|} \in H_{g}^{1}\left(B^{2}, S^{2}\right)$ is a continuous path in $H_{g}^{1}\left(B^{2}, S^{2}\right)$ joining $u^{k}(t=0)$ to $u^{k+1}(t=1)$, that is a homotopy between $u^{k}$ and $u^{k+1}$. Of course, the space $H_{g}^{1}\left(B^{2}, S^{2}\right)$ has the metric induced by the norm $\|\cdot\|_{H^{1}\left(B^{2}, \mathbb{R}^{3}\right)}$. Morevover it is clear that the map $u \rightarrow Q(u)$ defined in formula (1.1) is continuous with respect to the strong convergence in $H^{1}\left(B^{2}, \mathbb{R}^{3}\right)$. We deduce that the function

$$
\begin{aligned}
{[0,1] } & \rightarrow \mathbb{Z} \\
t & \rightarrow Q\left(\frac{u^{k}+t w^{k}}{\left|u^{k}+t w^{k}\right|}\right)-Q(\underline{u})
\end{aligned}
$$

is continuous with values in $\mathbb{Z}$ and therefore constant. 
Proposition 2.1. Let $u \in H_{g}^{1}\left(B^{2}, S^{2}\right)$. The map

$$
\begin{aligned}
\phi_{u}: T_{u} & \rightarrow H_{g}^{1}\left(B^{2}, S^{2}\right) \\
w & \rightarrow \frac{u+w}{|u+w|}
\end{aligned}
$$

is defined and continuous, where $H_{g}^{1}\left(B^{2}, S^{2}\right)$ has the metric induced by the norm $\|\cdot\|_{H^{1}\left(B^{2}, \mathbb{R}^{3}\right)}$. In particular, for $w \in T_{u}$ the map $[0,1] \ni t \rightarrow \frac{u+t w}{|u+t w|} \in H_{g}^{1}\left(B^{2}, S^{2}\right)$ is a homotopy between $u$ and $\frac{u+w}{|u+w|}$.

Proof. First notice that for $w \in T_{u}, \phi_{u}(w)=\frac{u+w}{\sqrt{1+|w|^{2}}}$, so that $\phi_{u}(w)$ is defined a.e. and $\left|\phi_{u}(w)(x)\right|=1$ a.e.

Second we show that $\tilde{\phi}_{u}: H^{1}\left(B^{2}, \mathbb{R}^{3}\right) \ni w \rightarrow \frac{u+w}{\sqrt{1+|w|^{2}}} \in H^{1}\left(B^{2}, \mathbb{R}^{3}\right)$ is defined and continuous. For $w \in$ $H^{1}\left(B^{2}, \mathbb{R}^{3}\right)$,

$$
\left|\frac{u(x)+w(x)}{\sqrt{1+|w(x)|^{2}}}\right| \leq \frac{1+|w(x)|}{\sqrt{1+|w(x)|^{2}}} \leq \sqrt{2} \quad \text { a.e. }
$$

so $\tilde{\phi}_{u}(w) \in L^{2}\left(B^{2}, \mathbb{R}^{3}\right)$. Let $i \in\{1,2\}$; from classical lemmas [4] on weak derivatives,

$$
\partial_{i} \tilde{\phi}_{u}(w)=\frac{\partial_{i} u+\partial_{i} w}{\left(1+|w|^{2}\right)^{\frac{1}{2}}}-\frac{(u+w)\left(\partial_{i} w \cdot w\right)}{\left(1+|w|^{2}\right)^{\frac{3}{2}}} .
$$

Then, using (2.4)

$$
\left|\partial_{i} \tilde{\phi}_{u}(w)(x)\right| \leq\left|\partial_{i} u(x)\right|+\left|\partial_{i} w(x)\right|+\sqrt{2}\left|\partial_{i} w(x)\right|,
$$

and $\partial_{i} \tilde{\phi}_{u}(w) \in L^{2}$. We have proved so far that $\tilde{\phi}_{u}(w) \in H^{1}\left(B^{2}, \mathbb{R}^{3}\right)$.

Now let $\left(w^{k}\right)$ be a sequence converging to $w$ in $H^{1}$. There exists a subsequence $\left(w^{k_{l}}\right)$ such that $w^{k_{l}}(x) \rightarrow w(x)$ a.e. We have $\tilde{\phi}_{u}\left(w^{k_{l}}\right)(x) \rightarrow \tilde{\phi}_{u}(w)(x)$ a.e. and $\left|\tilde{\phi}_{u}\left(w^{k_{l}}\right)(x)\right| \leq \sqrt{2}$ a.e. by (2.4), so by the Lebesgue dominated convergence theorem $\tilde{\phi}_{u}\left(w^{k_{l}}\right) \rightarrow \tilde{\phi}_{u}(w)$ in $L^{2}$. Since for every subsequence of $\tilde{\phi}_{u}\left(w^{k}\right)$ we can extract by the same means a subsequence converging to $\tilde{\phi}_{u}(w)$ in $L^{2}$, we obtain that the entire sequence converges to $\tilde{\phi}_{u}(w)$ in $L^{2}$.

Now let $i \in\{1,2\}$; there exists a subsequence $w^{k_{l}}$ and $f \in L^{2}$ such that $w^{k_{l}}(x) \rightarrow w(x)$ a.e., $\partial_{i} w^{k_{l}}(x) \rightarrow$ $\partial_{i} w(x)$ a.e. and $\left|\partial_{i} w^{k_{l}}(x)\right| \leq f(x)$ a.e. Then $\partial_{i} \tilde{\phi}_{u}\left(w^{k_{l}}\right)(x) \rightarrow \partial_{i} \tilde{\phi}_{u}(w)(x)$ a.e. and using (2.6),

$$
\left|\partial_{i} \tilde{\phi}_{u}\left(w^{k_{l}}\right)(x)\right| \leq\left|\partial_{i} u(x)\right|+3 f(x) \quad \text { a.e. }
$$

so by the Lebesgue dominated convergence theorem $\partial_{i} \tilde{\phi}_{u}\left(w^{k_{l}}\right) \rightarrow \partial_{i} \tilde{\phi}_{u}(w)$ in $L^{2}$. By unicity of the limit (same argument as above), we conclude that the entire sequence $\partial_{i} \tilde{\phi}_{u}\left(w^{k}\right)$ converges to $\partial_{i} \tilde{\phi}_{u}(w)$ in $L^{2}$. Summing up, we have proved that the sequence $\left(\tilde{\phi}_{u}\left(w^{k}\right)\right)$ converges to $\tilde{\phi}_{u}(w)$ in $H^{1}\left(B^{2}, \mathbb{R}^{3}\right)$ and that $\tilde{\phi}_{u}$ is continuous.

It is now easy to see that $\operatorname{tr}\left(\tilde{\phi}_{u}(w)\right)=\operatorname{tr}(u)$ for all $w \in H_{0}^{1}$, where $\operatorname{tr}$ denotes the trace of a $H^{1}$ function on $\partial B^{2}$. The equality is immediate if $w$ has a compact support in $B^{2}$ because in this case $\tilde{\phi}_{u}(w) \equiv u$ on a neighbourhood of $\partial B^{2}$. For $w \in H_{0}^{1}$ the equality comes by density of $\mathcal{C}_{c}^{\infty}$ into $H_{0}^{1}$ and by continuity of the trace and of $\tilde{\phi}_{u}$.

In conclusion we have shown that the map $\tilde{\phi}_{u}: H_{0}^{1} \rightarrow H_{g}^{1}\left(B^{2}, S^{2}\right)$ is continuous, and so is its restriction $\phi_{u}$ to $T_{u}$. 


\subsection{Discretization}

\subsubsection{Continuous $P^{1}$ elements}

We use continuous $P^{1}$ elements. Let $B^{2, h}$ be a polygon with vertices on $\partial B^{2}$ which is a discretization of $B^{2}$. Let $\mathcal{T}^{h}$ be an admissible triangulation (see [3]) of $B^{2, h}$. Let

$$
P^{1, h}:=\left\{u^{h} \in \mathcal{C}^{0}\left(B^{2, h}\right) \text { such that } u^{h} \text { is affine on every triangle } T_{i} \text { of } \mathcal{T}^{h}\right\}
$$

We denote by $N$ the number of nodes of the triangulation, $x_{1}, \ldots, x_{N}$ the nodes of the triangulation and $I_{b d} \subset\{1, \ldots, N\}$ the set of indices $i$ such that $x_{i}$ belongs to the boundary $\partial B^{2, h}$. We recall that $P^{1, h}$ is a subspace of $H^{1}\left(B^{2, h}, \mathbb{R}^{3}\right)$ of dimension $3 N$, an isomorphism to $\mathbb{R}^{3 N}$ being given by

$$
\begin{aligned}
P^{1, h} & \rightarrow\left(\mathbb{R}^{3}\right)^{N} \\
u^{h} & \rightarrow\left(u^{h}\left(x_{i}\right)\right)_{1 \leq i \leq N} .
\end{aligned}
$$

The discrete energy is defined for every $u \in H^{1}\left(B^{2, h}, \mathbb{R}^{3}\right)$ by

$$
E^{h}(u):=\frac{1}{2} \int_{B^{2, h}}|\nabla u|^{2} \mathrm{~d} x .
$$

This discrete energy is associated to the continuous symmetric bilinear form

$$
a^{h}(u, v):=\int_{B^{2, h}} \nabla u \cdot \nabla v \mathrm{~d} x \quad \forall u, v \in H^{1}\left(B^{2, h}, \mathbb{R}^{3}\right)
$$

Let us point out that there is a consistency error which comes from the approximation of $B^{2}$ by the polygon $B^{2, h}$; this consistancy error is again present in the energy where the integration is done on $B^{2, h}$ instead of $B^{2}$.

\subsubsection{Preservation of the degree}

The discretized version of $H_{g}^{1}\left(B^{2}, S^{2}\right)$ is

$$
\mathcal{E}_{g}^{h}:=\left\{u^{h} \in P^{1, h},\left|u^{h}\left(x_{i}\right)\right|=1 \forall i, u^{h}\left(x_{i}\right)=g\left(x_{i}\right) \forall i \in I_{b d}\right\} .
$$

Notice that $\mathcal{E}_{g}^{h}$ is not a subset of $H_{g}^{1}\left(B^{2}, S^{2}\right)$ since $\left|u^{h}(x)\right|<1$ in general if $x$ is not a node of the triangulation. However, if $u^{h} \in \mathcal{E}_{g}^{h}$ is a map that satisfies $u^{h}(x) \neq 0$ for all $x \in B^{2, h}$, then we can define the degree of $u^{h}$ as the degree of the map $\frac{u^{h}}{\left|u^{h}\right|}$ which is continuous with values in $S^{2}$ and imposed boundary values. Now with such a definition, the discrete energy is not in general greater than the discrete degree, yet this was a fundamental property in the theoretic study.

In the minimizing algorithm we fix a small $\delta>0$ and we choose a starting point $u^{h} \in \mathcal{E}_{g}^{h}$ such that $\left|u^{h}\left(x_{i}\right)-u^{h}\left(x_{j}\right)\right| \leq \delta$ for every edge $\left[x_{i}, x_{j}\right]$ of the triangulation: this ensures that $\left|u^{h}(x)\right| \geq 1-\varepsilon(\delta)$ for all $x \in B^{2, h}$, where $\varepsilon(\delta)$ tends to 0 when $\delta$ tends to 0 . In this case $u^{h}$ is a good approximation of a map with values in $S^{2}$ and its degree is well defined. In order to guarantee the preservation of the degree during the minimization, for every new $u^{h} \in \mathcal{E}_{g}^{h}$ that we get, we test whether $\left|u^{h}\left(x_{i}\right)-u^{h}\left(x_{j}\right)\right| \leq \delta$ for every edge $\left[x_{i}, x_{j}\right]$; if some edge $\left[x_{i}, x_{j}\right]$ does not satisfy this condition, then we divide in four each of the two triangles that share this edge.

We describe below this refinement procedure:

Algorithm 2.2 (Refinement).

Input Data. Choose

- a refinement angle $\delta$ small enough; 
- a triangulation $\mathcal{T}^{h}$ (nodes, triangles, edges) of $B^{2, h}$;

- a map $u^{h} \in \mathcal{E}_{g}^{h}$ such that $u^{h}(x) \neq 0$ for every $x \in B^{2, h}$.

Initial Data. Set $\mathcal{T}^{h^{\prime}}=\mathcal{T}^{h}$ and $u^{h^{\prime}}=u^{h}$.

Step 1: testing edges. Color in red every edge $\left[x_{i}, x_{j}\right]$ of $\mathcal{T}^{h^{\prime}}$ such that

$$
u^{h^{\prime}}\left(x_{i}\right) \cdot u^{h^{\prime}}\left(x_{j}\right)<\cos (\delta)
$$

If no edge is colored, stop the refinement algorithm.

Step 2: nodes. For every red edge, find the 2 triangles $T_{i}$ and $T_{j}$ that share this edge (1 triangle $T_{i}$ if it is a boundary edge), and color in red the edges of $T_{i}$ and $T_{j}$ (of $\left.T_{i}\right)$.

Add to the nodes of the triangulation $\mathcal{T}^{h^{\prime}}$ the middle point of every red edge.

Step 3: triangles and edges. Divide each triangle $T_{i}$ of $\mathcal{T}^{h^{\prime}}$ in 4, 3, 2, or 1 according to the number 3, 2, 1, or 0 of colored edges that $T_{i}$ has. Change and add the corresponding edges of $\mathcal{T}^{h^{\prime}}$ according to this division.

Step 4: interpolation. Let $u^{h^{\prime \prime}}$ be the map in $P^{1, h^{\prime}}$ such that $u^{h^{\prime \prime}}\left(x_{i}^{\prime}\right)=\frac{u^{h^{\prime}}\left(x_{i}^{\prime}\right)}{\left|u^{h^{\prime}}\left(x_{i}^{\prime}\right)\right|}$ for every node $x_{i}^{\prime}$ of the new triangulation $\mathcal{T}^{h^{\prime}}$.

Replace $u^{h^{\prime}}$ by $u^{h^{\prime \prime}}$ and go to step 1 .

\section{Output Data.}

- A triangulation $\mathcal{T}^{h^{\prime}}$ of $B^{2, h}$ which is a subtriangulation of $\mathcal{T}^{h}$.

- The interpolation $u^{h^{\prime}}$ of $u^{h}$ on $\mathcal{T}^{h^{\prime}}$ that satisfies $u^{h^{\prime}}\left(x_{i}\right) \cdot u^{h^{\prime}}\left(x_{j}\right) \geq \cos (\delta)$ for every edge $\left[x_{i}, x_{j}\right]$ of the triangulation $\mathcal{T}^{h^{\prime}}$.

\section{Remarks.}

1. The output $u^{h^{\prime}}$ has the same degree as the input $u^{h}$.

2. The refinement procedure converges in a finite (small, usually 1) number of iterations.

\subsubsection{Discrete algorithm}

Using the identification $P^{1, h} \equiv\left(\mathbb{R}^{3}\right)^{N}$ defined above (2.7), it is easy to see $\mathcal{E}_{g}^{h}$ as a submanifold of $\mathbb{R}^{3}$. The tangent space $T_{u^{h}}^{h}$ at point $u^{h} \in \mathcal{E}_{g}^{h}$ is obtained by linearization of the constraints:

$$
T_{u^{h}}^{h}:=\left\{w^{h} \in P^{1, h}, w^{h}\left(x_{i}\right) \cdot u^{h}\left(x_{i}\right)=0 \forall i, w^{h}\left(x_{i}\right)=0 \forall i \in I_{b d}\right\} .
$$

$T_{u^{h}}^{h}$ is the discretized version of $T_{u}$.

The discrete algorithm has the same structure as in the continuous case plus the refinement technique.

Algorithm 2.3 (SG/CG for discrete harmonic maps).

\section{Initial Data.}

- Let $\mathcal{T}^{h}$ be a triangulation of $B^{2, h}$.

- Choose a refinement angle $\delta>0$ small enough.

- Set $k=0$ and choose $u^{h, k} \in \mathcal{E}_{g}^{h}$ with given degree.

- Refinement with respect to $\delta$. Replace $h$ by $h^{\prime}$.

Step 1. Find $w^{h, k} \in T_{u^{h, k}}^{h}$ such that

$$
a^{h}\left(w^{h, k}, \varphi\right)=a^{h}\left(u^{h, k}, \varphi\right) \quad \forall \varphi \in T_{u^{h, k}}^{h} .
$$

Step 2. Let $u^{h, k+1}$ be the map in $P^{1, h}$ such that

$$
u^{h, k+1}\left(x_{i}\right)=\frac{u^{h, k}-w^{h, k}}{\left|u^{h, k}-w^{h, k}\right|}\left(x_{i}\right) \quad \text { for } i=1,2 \ldots, N .
$$


Step 3. Refinement with respect to $\delta$. Replace $h$ by $h^{\prime}$.

Replace $k$ by $k+1$ and go to step 1 .

\section{Remarks.}

1. After each refinement step, the finite element stiffness matrix has to be computed.

2. The difficult step $\mathbf{1}$ is solved using a conjugate gradient method [1].

3. We are not able to prove that the degree of the map $u^{h, k+1}$ computed in step $\mathbf{2}$ is the same as the degree of $u^{h, k}$. If $\frac{u^{h, k}-t w^{h, k}}{\left|u^{h, k}-t w^{h, k}\right|}(x) \neq 0$ for all $x \in B^{2, h}$ and all $t \in[0,1]$, then the map

$$
\begin{aligned}
{[0,1] } & \rightarrow \mathcal{C}^{0}\left(B^{2, h}, \mathbb{R}^{3}-\{0\}\right) \\
t & \rightarrow \frac{u^{h, k}-t w^{h, k}}{\left|u^{h, k}-t w^{h, k}\right|}
\end{aligned}
$$

is a homotopy with fixed boundary values between $u^{h, k}$ and $u^{h, k+1}$ : in this case the degrees are the same. Otherwise, the degree changes. So we should actually check this and stop the algorithm if a problem occurs. In practice, this never happens with this algorithm, because $\left|w^{h, k}\left(x_{i}\right)\right|$ is small.

However, in the case of the Newton Algorithm 3.1, this situation sometimes occured (because of the instability of Newton). In those cases we used a visual check to see the problem!

4. The convergence of such an algorithm is difficult to prove. For given $\delta>0$ and initial triangulation, if the refinement stops after a certain iteration, then the discrete energy decreases and we have a convergence result similar to the Theorem 2.1: the sequence $\left(u^{h, k}\right)_{k}$ converges (up to a subsequence) to a critical point of $E^{h}$ in $\mathcal{E}_{g}^{h}$. But in some cases (the singular ones) the refinement never stops.

Another open question would be to prove that the discrete solution converges to a smooth harmonic map (for the regular cases).

\subsection{Numerical results}

\subsubsection{Starting point}

For the computation, we need to find the starting map $u^{0}$.

Since the boundary condition $g$ is of class $\mathcal{C}^{1}, g\left(\partial B^{2}\right)$ is of (Hausdorff) dimension 1 at most, there exists a point $P \in S^{2}$ such that $P \notin g\left(\partial B^{2}\right)$. We suppose in the following that the north pole is such a point. Let $P_{S}:=-P$ be the south pole, then we can define a homotopy between $g$ and the south pole,

$$
\begin{aligned}
& F_{g}:[0,1] \quad \times \quad \partial B^{2} \rightarrow S^{2} \\
& (t \quad, \quad z) \rightarrow \frac{(1-t) P_{S}+t g(z)}{\left|(1-t) P_{S}+t g(z)\right|},
\end{aligned}
$$

which belongs to $\mathcal{C}^{1}\left([0,1] \times \partial B^{2}\right)$.

We also define for a given degree $n \in \mathbb{Z}, v^{n}: B^{2}\left(0, \frac{1}{2}\right) \rightarrow \mathbb{C}$ by

$$
v^{n}(z)= \begin{cases}P_{S} & \text { if } n=0 \\ (\tan (\pi|z|) z /|z|)^{n} & \text { if } n>0 \\ (\tan (\pi|\bar{z}|) \bar{z} /|\bar{z}|)^{|n|} & \text { if } n<0\end{cases}
$$

which covers $n$ times the sphere $S^{2}$. Let finally $\Pi_{S}$ denote the stereographic projection from the south pole on the equator plane $\mathbb{R}^{2}$. Then

$$
\begin{aligned}
\Pi_{S}^{-1}: \mathbb{R}^{2} & \rightarrow \\
(x, y) & \rightarrow\left(\frac{2 x}{1+x^{2}+y^{2}}, \frac{S^{2} \subset \mathbb{R}^{3}}{1+x^{2}+y^{2}}, \frac{1-\left(x^{2}+y^{2}\right)}{1+x^{2}+y^{2}}\right)
\end{aligned}
$$




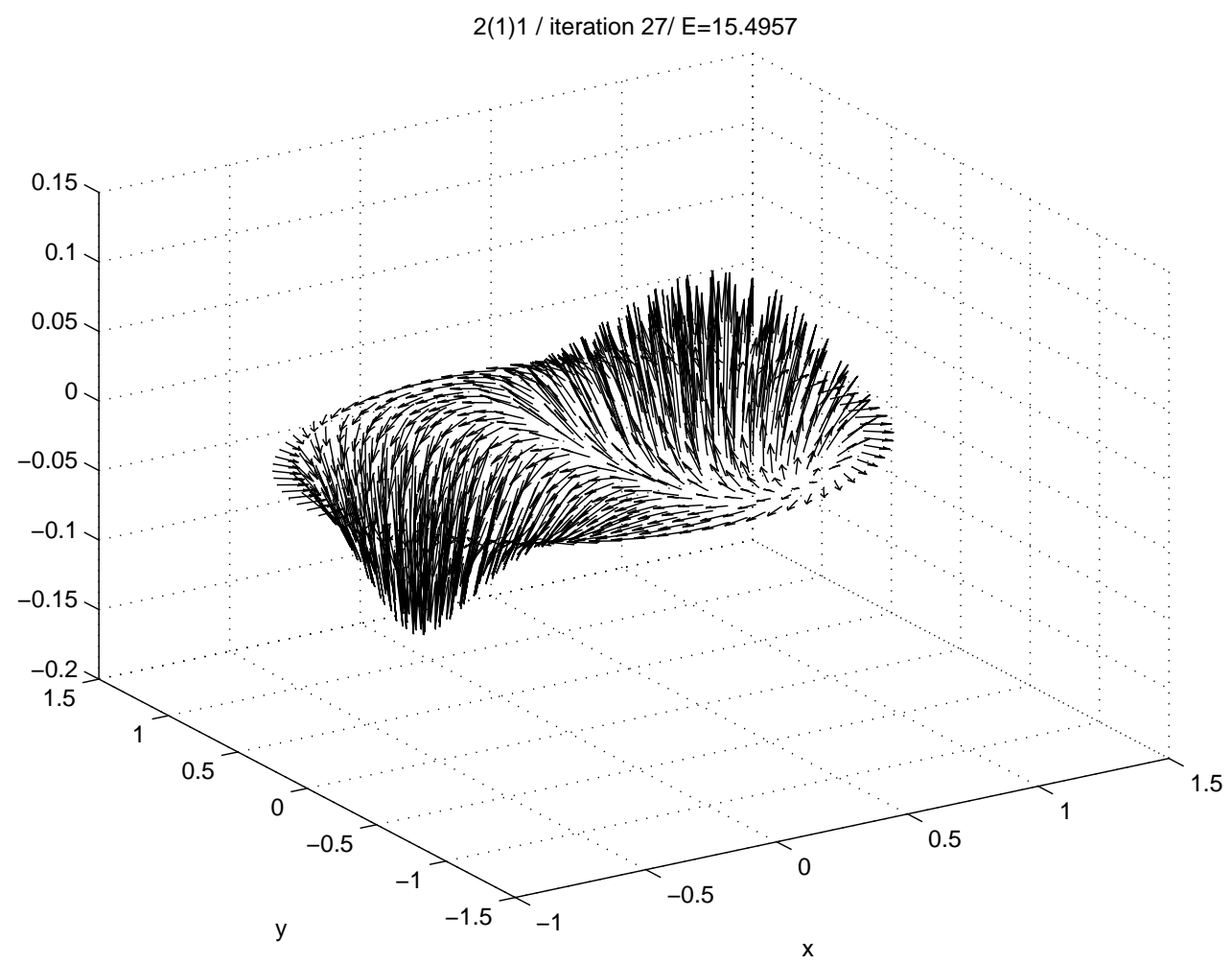

Figure 1. SG/CG for 2(1)1 (rescaled).

The reference map $\underline{u}$ chosen is $\underline{u}(z)=F_{g}\left(|z|, \frac{z}{|z|}\right)$. The initial map of degree $n \in \mathbb{Z}$ is

$$
u^{0}(z)= \begin{cases}\Pi_{S}^{-1}\left(v^{n}(z)\right) & |z|<\frac{1}{2} \\ F_{g}\left(2\left(|z|-\frac{1}{2}\right), \frac{z}{|z|}\right) & \frac{1}{2} \leq|z| \leq 1\end{cases}
$$

Remark. it is clear that $u^{0}$ is continuous on $B^{2}$ and has the degree $n$. For the algorithm 2.3 , we actually use as a starting point the $P_{1}$ interpolate of $u^{0}$ on the mesh $\mathcal{T}^{h}$, which clearly belongs to $H^{1}\left(B^{2, h}, \mathbb{R}^{3}\right)$.

\subsubsection{Numerical results}

The boundary condition $g$ is of the type $g(z)=\Pi_{S}\left(a z^{n}\right)$. With this choice, we know according to Soyeur [30] that the minimum in the class $\mathcal{E}_{g, 0}$ is $\Pi_{S}\left(\frac{a}{\bar{z}^{n}}\right)$, that the minimum in the class $\mathcal{E}_{g, n}$ is $\Pi_{S}\left(a z^{n}\right)$, and that the only classes that admit a minimum are $\mathcal{E}_{g, 0}, \widetilde{\mathcal{E}}_{g, 1}, \ldots, \mathcal{E}_{g, n}$. The minima in the intermediate classes $\mathcal{E}_{g, 1}, \mathcal{E}_{g, 2}, \ldots$, $\mathcal{E}_{g, n-1}$ are not known explicitly.

We use a triplet $n(a) p$ to indicate that we have computed the minimizer in $\mathcal{E}_{g, p}$ with $g(z)=\Pi_{S}\left(a z^{n}\right)$.

If the figures shown we have set $\delta=\pi / 8$. The stopping test is that the refinement has stopped since at least 20 iterations and $\left|E\left(u^{k+1}\right)-E\left(u^{k}\right)\right| \leq 10^{-5}$. 


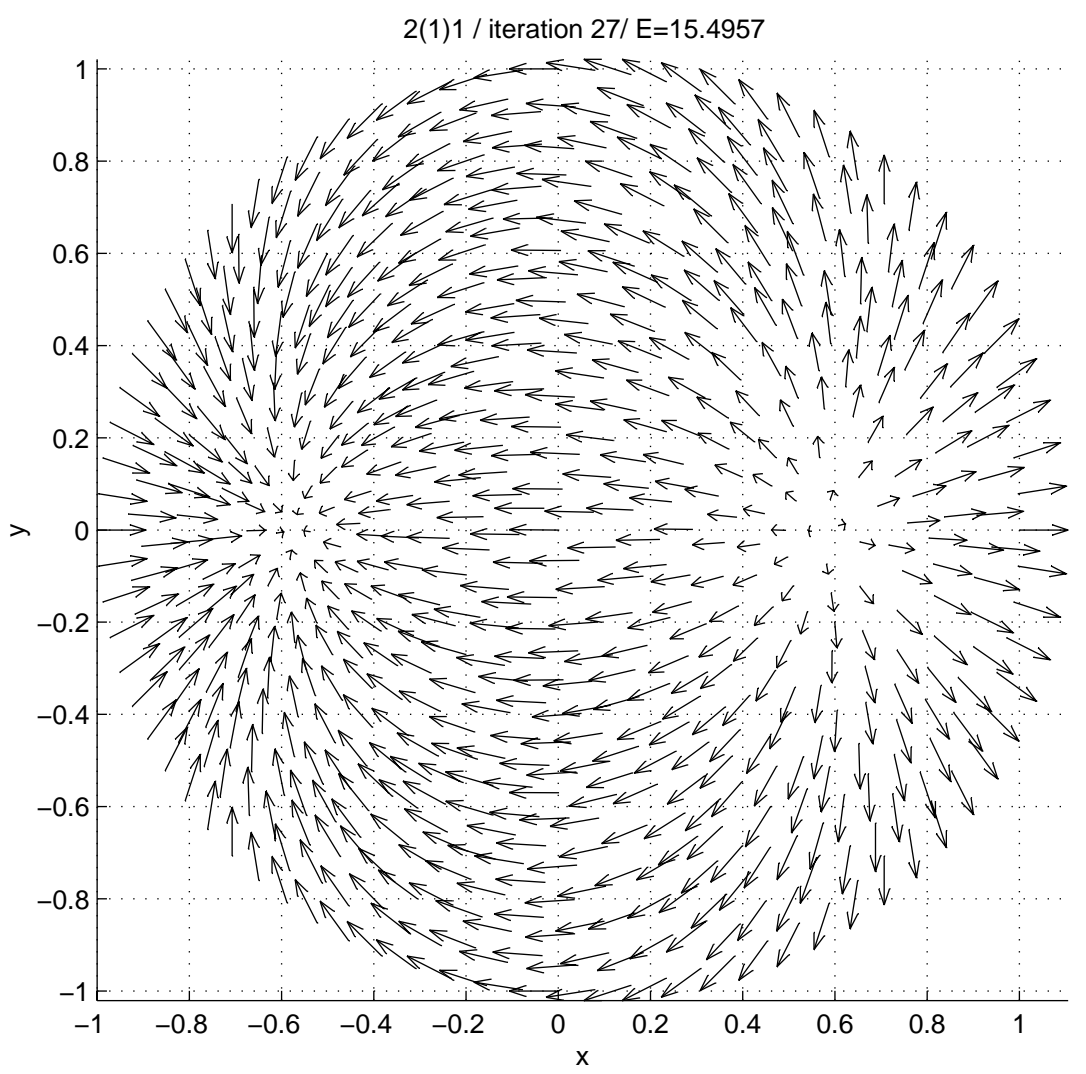

FIGURE 2. SG/CG for 2(1)1.

Harmonics maps. Figure 1 shows the minimizer in $\mathcal{E}_{g, 1}$ for $g(z)=z^{2}$ (with rescaled axes): the rescaling points out that this surface covers +1 time the north hemisphere and -1 time the south hemisphere (actually, the degree can be simply computed by the number of north hemisphere that are covered). The symetries of this map can be observed on its projection on the $x O y$ plan in Figure 2 (with normalized axes). The solution has the same symetry around the axe $O x$ than the boundary condition.

Figure 3 shows (the projection on the $x O y$ plan of the minimizer in $\mathcal{E}_{g, 1}$ for $g(z)=z^{3}$ : it covers +1 time the north hemisphere and -2 times the south hemisphere. It is interesting to notice that the boundary condition has 2 symetry axes $O x$ and $O y$ whereas the solution only has the symetry axe $O x$. This indicates that we could obtain another harmonic map by minimizing $E$ among maps in $\mathcal{E}_{g, 1}$ which have the symetry of the boundary.

Figure 4 show the minimizer in $\mathcal{E}_{g, 2}$ with the same boundary condition $g(z)=z^{3}$, which covers +2 times the north hemisphere and -1 time the south. Its projection on the $x O y$ plan in Figure 5 is identical to the previous one.

It is worth noticing that (because of the starting point for the algorithm) this latter map requires more computation than the previous one (it has actually been computed by a conjugate gradient algorithm, see Sect. 4). In Figure 6 the final mesh for the computation of this map 3(1)1 is shown. This mesh shows the history of the computation: the larger triangles stand for the initial mesh; the fine refinement in the center and around a circle of radius $\sim 3 / 4$ were made for the starting map. The coverging of the south pole in the final solution can be seen a litlle bit in the intermediate refinement around thepoint $(-0.8,0)$. 


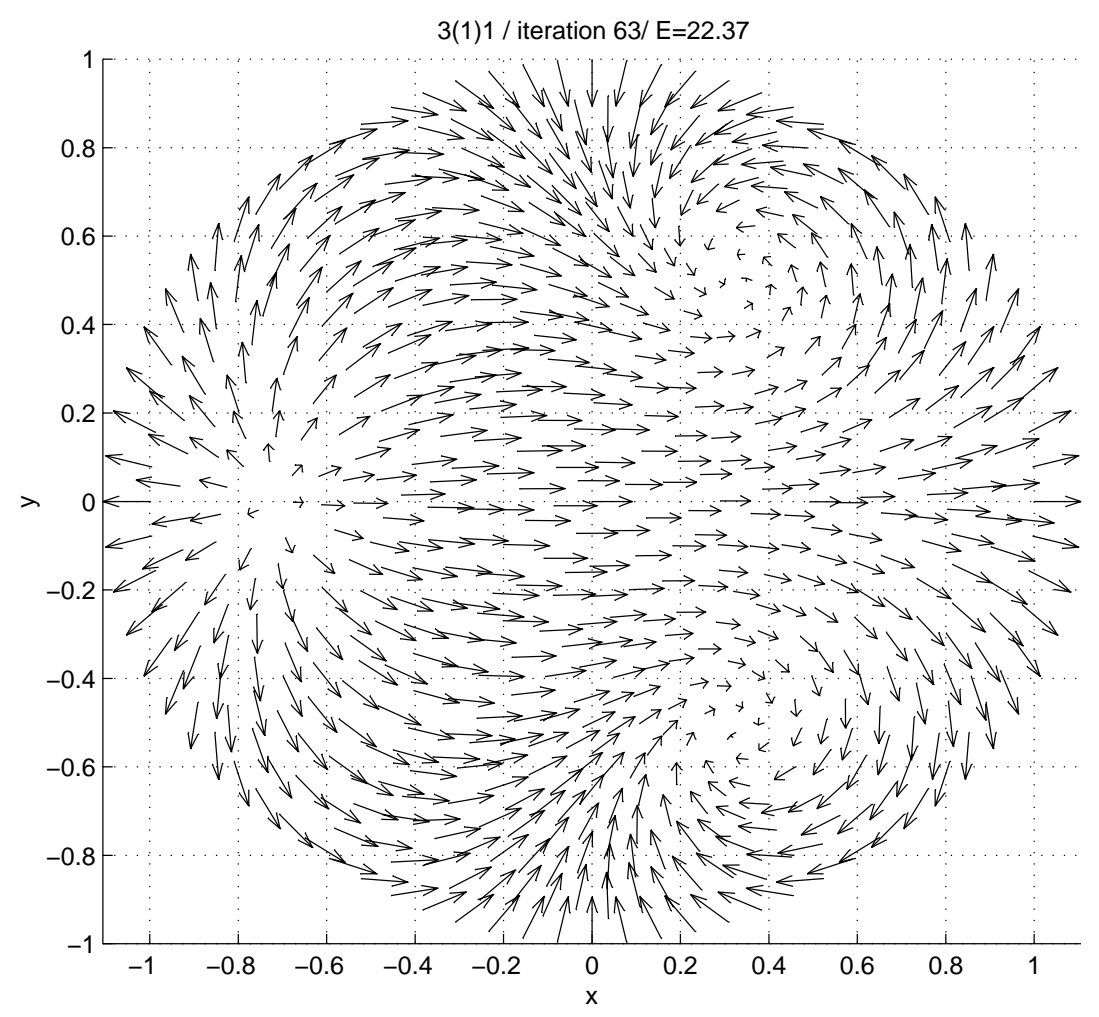

Figure 3. SG/CG for 3(1)1.

Limits of the algorithm. We explain some general features of the Sobolev gradient Algorithm 2.3 for a given $n \geq 1$ and $a \geq 1$ :

- The minimizer in $\mathcal{E}_{g, 0}$, which is also the absolute minimizer, is computed with few (usually less than 20) iterations of the algorithm and without refinement;

- For a degree $p \neq 0$, without the refinement procedure, the algorithm converges in most cases to the absolute minimizer found above;

- With the refinement procedure and for a degree $p \in\{1, \ldots, n\}$, the refinement stops after a certain iteration $k_{0}$ and the algorithm converges to the (discrete) minimizer in $\mathcal{E}_{p}$ in more iteration than for the degree 0 (from 20 to 2000, depending mainly on the strong gradient of the final solution and its localisation);

- For a degree $p \notin\{1, \ldots, n\}$, the refinement procedure never stops.

\section{NEWTON ALGORITHM FOR HARMONIC MAPS}

In this section we first remind that the metric space $H_{g}^{1}\left(B^{2}, S^{2}\right)$ fails to be a submanifold of $H^{1}\left(B^{2}, \mathbb{R}^{3}\right)$. However, in the discrete case $\mathcal{E}_{g}^{h}$ is clearly a submanifold of $P^{1, h}$ and we can apply the Newton algorithm for manifolds 5.1. The idea of this algorithm is to write the classical Newton algorithm for Banach spaces [7] by using a local chart which approaches well the Riemannian structure of the manifold. Here we are able to do the numerical computation, which in the general case is too complexe, because we deal with $S^{2}$ and the local charts are trivial. The hope is to improve the convergence of the Sobolev gradient Algorithm 2.3. We comment the numerical results. 


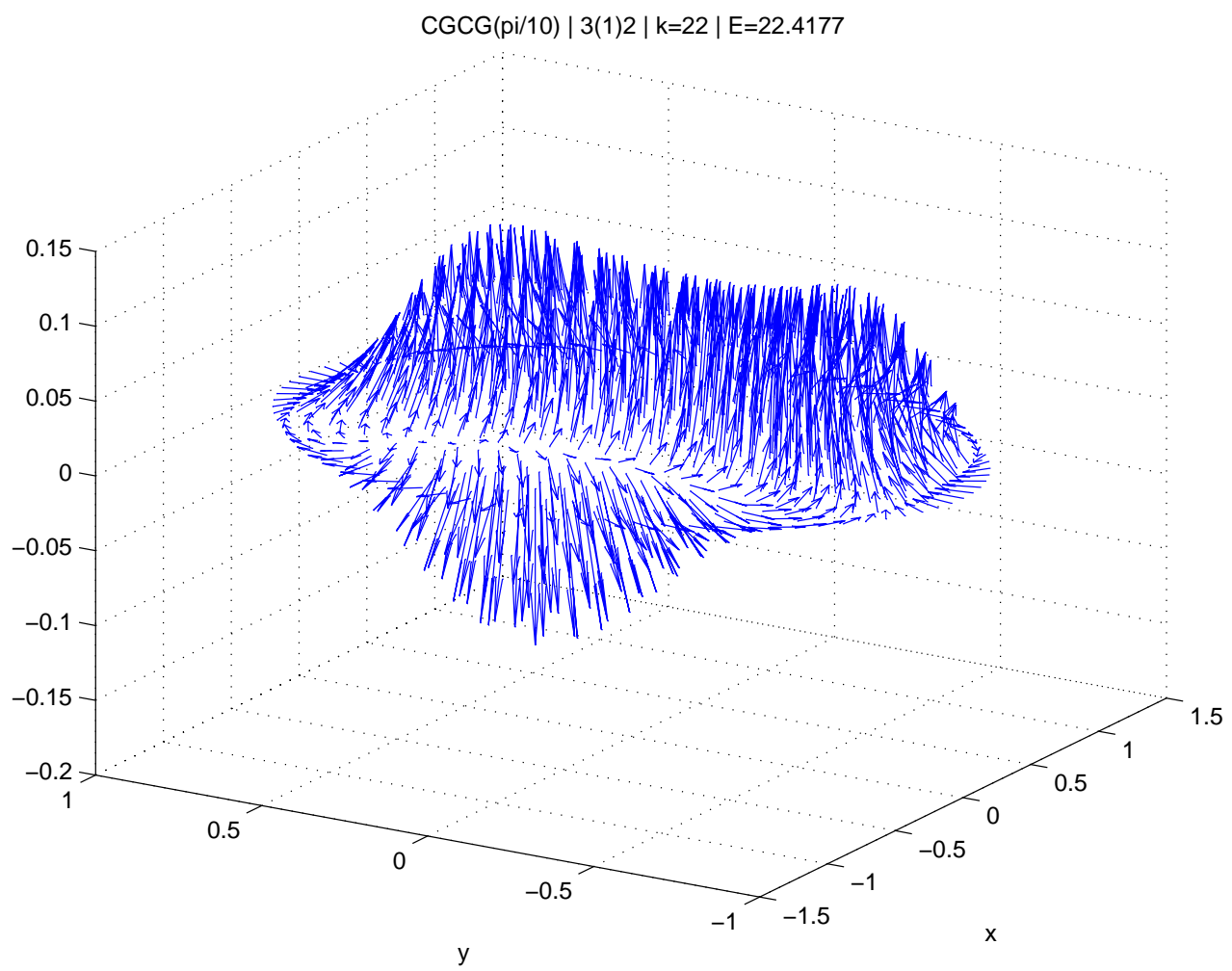

Figure 4. CG/CG for 3(1)2 (rescaled).

\subsection{Remark on the structure of $H_{g}^{1}\left(B^{2}, S^{2}\right)$}

Let $m \geq 0$ be an integer. Define as in the introduction

$$
H_{g}^{1}\left(B^{m}, S^{2}\right):=\left\{u \in H^{1}\left(B^{M}, \mathbb{R}^{3}\right),|u(x)|=1 \text { a.e., } u_{\mid \partial B^{m}}=g_{\mid \partial B^{m}}\right\}
$$

where $B^{m}:=\left\{x \in \mathbb{R}^{m},|x|<1\right\}$ is the unit ball in $\mathbb{R}^{m}$ and $g$ is the boundary condition which belongs to $\mathcal{C}^{1}\left(\overline{B^{m}}, S^{2}\right)$ so that $H_{g}^{1}\left(B^{m}, S^{2}\right)$ is not empty .

If $m=1$, then $H_{g}^{1}\left(B^{m}, S^{2}\right)$ is a submanifold of $H^{1}\left(B^{m}, \mathbb{R}^{3}\right)$ of infinite dimension, modelled on the (unique) separable Hilbert space $l^{2}$. This is a well known fact used in the theory of geodesics (see [17]), especially when we deal with a general surface of $\mathbb{R}^{3}$ instead of $S^{2}$. For $u \in H_{g}^{1}\left(B^{1}, S^{2}\right)$ the tangent space is

$$
T_{u}:=\left\{w \in H_{0}^{1}\left(B^{1}, \mathbb{R}^{3}\right) \text { such that } w(x) \cdot u(x)=0 \text { a.e. }\right\},
$$

and a local chart is given by:

$$
\begin{aligned}
\phi_{u}: T_{u} & \rightarrow H_{g}^{1}\left(B^{1}, S^{2}\right) \\
w & \rightarrow \frac{u+w}{|u+w|}
\end{aligned}
$$

One key point in the proof is the Sobolev imbedding $H^{1}\left(B^{1}\right) \subset \mathcal{C}^{0}\left(\overline{B^{1}}\right)$. 


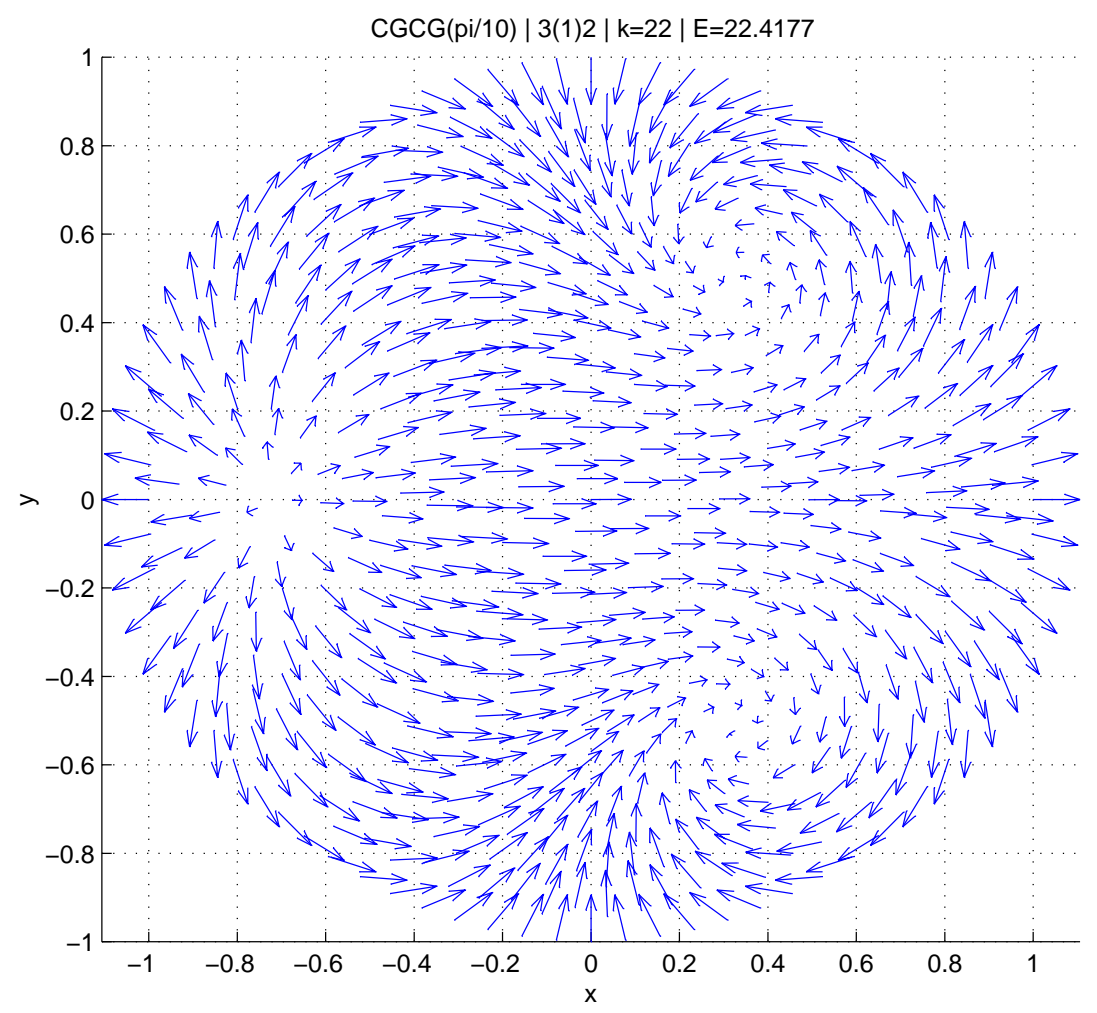

Figure 5. CG/CG for 3(1)2.

If $m \geq 2$ then we can still define the space $T_{u}$ (as we did in formula (2.1) for $m=2$ ) and the map $\phi_{u}$ but the Sobolev imbedding is no longer valid and $H_{g}^{1}\left(B^{m}, S^{2}\right)$ has no differentiable structure (see [15]). The reason for which $\phi_{u}$ fails to be a local chart is because it is not locally surjective.

\subsection{The Newton/CG algorithm for discrete harmonic maps}

Unlike $H_{g}^{1}\left(B^{2}, S^{2}\right)$, its discrete version $\mathcal{E}_{g}^{h}$ does have a differentiable structure because of the finite dimension: $\mathcal{E}_{g}^{h}$ is a submanifold of $P^{1, h}$. In order to compute discrete harmonic maps we can thus use a Newton algorithm for manifolds 5.1 as described in the appendix.

In the following we use notations of Section 2.2. We make a constant use of the identification $P^{1, h} \equiv\left(\mathbb{R}^{3}\right)^{N}$ and of the shortcut $u_{i}=u^{h}\left(x_{i}\right)$ for an element $u^{h} \in \mathcal{E}_{g}^{h}$ and a node $x_{i}$ of the triangulation. We remind that $I_{b d} \subset\{1, \ldots, N\}$ is the set of indices $i$ such that $x_{i}$ belongs to the boundary $\partial B^{2, h}$.

We see this time $\mathcal{E}_{g}^{h}$ as a submanifold of $\mathbb{R}^{3 N}$ (rather than a subspace of $P^{1, h}$ ),

$$
\mathcal{E}_{g}^{h}:=\left\{\left(u_{i}\right)_{1 \leq i \leq N} \in\left(\mathbb{R}^{3}\right)^{N},\left|u_{i}\right|=1 \forall i, u_{i}=g\left(x_{i}\right) \forall i \in I_{b d}\right\}
$$

and the tangent space is

$$
T_{u^{h}}^{h}:=\left\{\left(w_{i}\right) \in\left(\mathbb{R}^{3}\right)^{N}, w_{i} \cdot u_{i}=0 \forall i, w_{i}=0 \forall i \in I_{b d}\right\}
$$




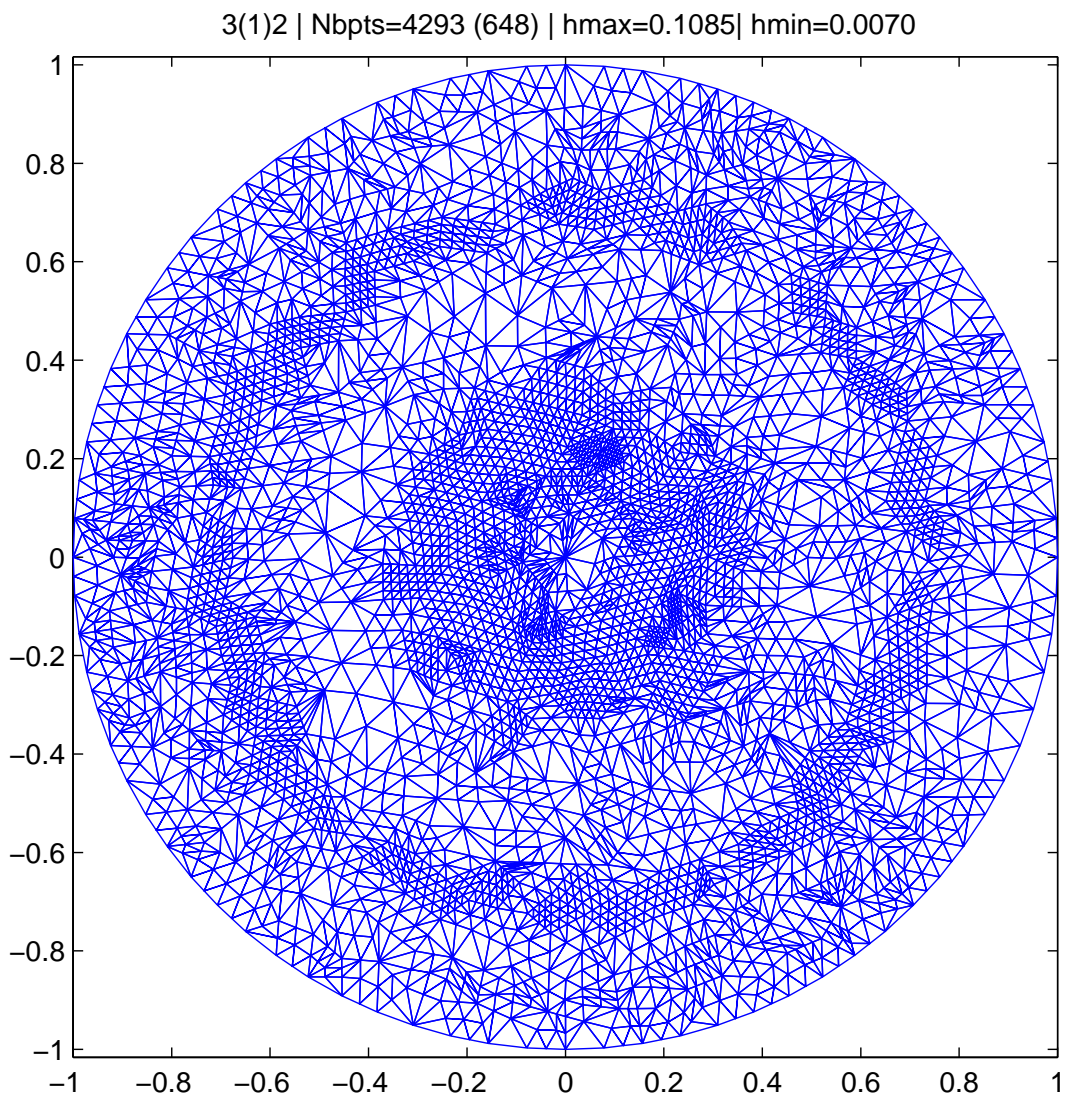

Figure 6. CG/CG for 3(1)2.

It is easy to see that for every $u^{h} \in \mathcal{E}_{g}^{h},\left(T_{u^{h}}^{h}, \phi_{u^{h}}^{h}\right)$ is a local chart that satisfies Assumptions 5.1, where

$$
\begin{aligned}
\phi_{u^{h}}^{h}: \quad T_{u^{h}}^{h} & \rightarrow \mathcal{E}_{g}^{h} \\
\left(w_{i}\right)_{1 \leq i \leq N} & \rightarrow\left(\frac{u_{i}+w_{i}}{\left|u_{i}+w_{i}\right|}\right)_{1 \leq i \leq N} .
\end{aligned}
$$

We want to find critical points of the energy $E^{h}\left(u^{h}\right)=\frac{1}{2} a^{h}\left(u^{h}, u^{h}\right)$ on the submanifold $\mathcal{E}_{g}^{h} \subset \mathbb{R}^{3 N}$. Since $E^{h}$ is a continuous quadratic form on $\mathbb{R}^{3 N}$ it is smooth on $\mathbb{R}^{3 N}$. As a consequence the map $J_{u^{h}}^{h}:=E^{h} \circ \phi_{u^{h}}^{h}$ is smooth on $T_{u^{h}}^{h}$. For $i \in\{1, \ldots, N\}$

$$
\frac{u_{i}+w_{i}}{\sqrt{1+\left|w_{i}\right|^{2}}}=u_{i}+w_{i}-\frac{u_{i}}{2}\left|w_{i}\right|^{2}+o\left(\left|w_{i}\right|^{2}\right)
$$

so we find:

$$
\begin{aligned}
J_{u^{h}}^{h \prime}(0) \cdot w^{h} & =a^{h}\left(u^{h}, w^{h}\right) \\
J_{u^{h}}^{h \prime \prime}(0)\left\langle w^{h}, w^{h}\right\rangle & =a^{h}\left(w^{h}, w^{h}\right)-b_{u^{h}}^{h}\left(w^{h}, w^{h}\right)
\end{aligned}
$$


for all $w^{h} \in T_{u^{h}}^{h}$. Here $b_{u^{h}}^{h}(\cdot, \cdot)$ is the bilinear symetric form such that

$$
b_{u^{h}}^{h}\left(w^{h}, w^{h}\right)=a^{h}\left(u^{h}, u^{h}\left|w^{h}\right|^{2}\right) \quad \forall w^{h} \in T_{u^{h}}^{h},
$$

where $u^{h}\left|w^{h}\right|^{2}$ denotes the element $\left(u_{i}\left|w_{i}\right|^{2}\right)_{1 \leq i \leq N}$ in $\left(\mathbb{R}^{3}\right)^{N} \equiv P^{1, h}$.

For the Newton algorithm we use the same refining strategy 2.2 as in the Algorithm 2.3.

Algorithm 3.1 (Newton/CG for discrete harmonic maps).

Initial Data.

- Let $\mathcal{T}^{h}$ be a triangulation of $B^{2, h}$;

- Choose a refinement angle $\delta>0$ small enough;

- Set $k=0$ and choose $u^{h, k} \in \mathcal{E}_{g}^{h}$ with given degree;

- Refinement with respect to $\delta$. Replace $h$ by $h^{\prime}$.

Step 1. Find $w^{h, k} \in T_{u^{h, k}}^{h}$ such that

$$
J_{u^{h}}^{h \prime \prime}(0)\left\langle w^{h, k}, \varphi\right\rangle=-J_{u^{h}}^{h \prime}(0) \cdot \varphi \quad \forall \varphi \in T_{u^{h, k}}^{h} .
$$

Step 2. Let $u^{h, k+1}$ be the map in $P^{1, h}$ such that

$$
u^{h, k+1}\left(x_{i}\right)=\frac{u^{h, k}+w^{h, k}}{\left|u^{h, k}+w^{h, k}\right|}\left(x_{i}\right) \text { for } i=1,2 \ldots, N .
$$

Step 3 Refinement with respect to $\delta$. Replace $h$ by $h^{\prime}$.

Replace $k$ by $k+1$ and go to step 1 .

\subsection{Numerical results}

We used the same boundary condition and initial value as in Section 2.3 (a test-case in given by a triplet $n(a) p)$. Here follows the conclusions of the tests:

- The Newton Algorithm 3.1 computes the minimizer in $\mathcal{E}^{0}$ in very few iterations (less than 10) and the convergence is quadratic. But this case in not really interesting since the Sobolev gradient algorithm was satisfactory and great precision is not required;

- For a degree $p \in\{1, \ldots, N\}$ the Newton algorithm does not work in general: either it converges to the absolute minimizer by changing homotopy class (the map $u^{h, k+1}$ computed in step 2 does not have the same degree as $u^{h, k}$, see the remarks following Algorithm 2.3), either it exploses quickly;

- Nevertheless in Figure 7 that compares the convergence-rates we have used the test-case 1(3)1 for which the Newton algorithm converges. We clearly see the quadratic convergence.

In conclusion the Newton algorithm has the usual advantages and drawbacks of the Newton method: when it converges, the convergence is fast, otherwise it exploses or converges to another critical point than expected. The convergence is only local: it is garanteed if the starting point is close enough from the solution. Another algorithm is needed in order to guarantee both global and fast convergence: that is the conjugate gradient.

\section{Conjugate Gradient For harmonic MAPS}

In this section we write the conjugate gradient algorithm for manifolds 6.2 in the case of discrete harmonic maps. The idea of this algorithm is to write the classical (nonlinear) conjugate gradient [29] by using a local chart which approaches well the Riemannian structure of the manifold. As in the Newton algorithm, we are able to do the numerical computation (which for a general manifold is too complexe) because we deal with $S^{2}$ and the local charts are trivial. The generalization is more complexe than the Newton algorithm since in order to do the computation at the current iteration, we need some information from the former iteration. We comment the numerical results. 


\subsection{Conjugate gradient/CG}

We have already seen that $\mathcal{E}_{g}^{h}$ (the discrete version of $H_{g}^{1}\left(B^{2}, S^{2}\right)$ ) is a submanifold of $\mathbb{R}^{3 N}$. We write the algorithm given in Appendix 6.2 in this case, using the refining strategy 2.2. We use notations of Section 3.2. The integer $N_{b d}$ is the number of nodes in the boundary.

Algorithm 4.1 (CG/CG for discrete harmonic maps).

\section{Initial Data.}

- Let $\mathcal{T}^{h}$ be a triangulation of $B^{2, h}$.

- Choose a refinement angle $\delta>0$.

- Set $k=0$ and choose $u^{h, k} \in \mathcal{E}_{g}^{h}$ with given degree.

- Refinement with respect to $\delta$. Replace $h$ by $h^{\prime}$.

- Choose an integer $l_{\max } \leq 2 N-2 N_{b d}$ and set $l=0$.

Step 1. Find $r^{h, l} \in T_{u^{h, k}}^{h}$ such that

$$
a^{h}\left(r^{h, l}, \varphi\right)=a^{h}\left(u^{h, k}, \varphi\right) \quad \forall \varphi \in T_{u^{h, k}}^{h}
$$

Step 2. If $l \geq 1$ let $\bar{d}^{h, l-1}=\left(\bar{d}_{i}^{l-1}\right)_{1 \leq i \leq N}$ be the map in $T_{u^{h, k}}$ defined by:

$$
\bar{d}_{i}^{l-1}= \begin{cases}-\frac{d_{i}^{l-1} \cdot w_{i}^{l-1}}{\sqrt{1+\left|w_{i}^{l-1}\right|^{2}}} u_{i}^{k-1}+\frac{d_{i}^{l-1} \cdot w_{i}^{l-1}}{\sqrt{1+\left|w_{i}^{l-1}\right|^{2}} \frac{w_{i}^{l-1}}{\left|w_{i}^{l-1}\right|^{2}}} & \text { if } w_{i}^{l-1} \neq 0 \\ 0 & \text { if } w_{i}^{l-1}=0\end{cases}
$$

for all $i \in\{1, \ldots, N\}$.

Step 3. If $l=0$ set $d^{h, l}=r^{h, l}$, else set

$$
d^{h, l}=r^{h, l}+\frac{a^{h}\left(r^{h, l}, r^{h, l}\right)}{a^{h}\left(r^{h, l-1}, r^{h, l-1}\right)} \bar{d}^{h, l-1} .
$$

Step 4. If $a^{h}\left(r^{h, l}, d^{h, l}\right) \leq 0$ set $l=0$ and $d^{h, l}=r^{h, l}$.

Step 5. Set

$$
\mu^{l}=-\frac{a^{h}\left(r^{h, l}, d^{h, l}\right)}{J_{u^{h}}^{h \prime}(0)\left\langle d^{h, l}, d^{h, l}\right\rangle} .
$$

Step 6. Set $w^{h, l}=\mu^{l} d^{h, l}$ and let $u^{h, k+1}=\left(u_{i}^{k+1}\right)_{1 \leq i \leq N}$ be the map in $P^{1, h}$ such that

$$
u_{i}^{h, k+1}=\frac{u_{i}^{h, k}+w_{i}^{h, l}}{\left|u_{i}^{h, k}+w_{i}^{h, l}\right|} \quad \forall i \in\{1, \ldots, N\} .
$$

Step 7.

- Refinement with respect to $\delta$. Replace $h$ by $h^{\prime}$.

- If $l=l_{\max }$ set $l=0$.

- Replace $l$ by $l+1, k$ by $k+1$ and go to step 1 (until convergence).

Remarks.

1. The refining method used here is a slight modification of the method 2.2. For the computation of the old direction $\bar{d}^{h, l-1}$ in step 2 at iteration $l \geq 1$, we need the vectors $u^{h, k-1}, d^{h, l-1}$ and $w^{h, l-1}$ of the iteration $l-1$ on the refined triangulation. This is done by interpolation in the same way that we have interpolated $u^{h, l}$ on every new node. 
2. The optimal step-size $u^{l}$ in step $\mathbf{5}$ is computed with only one iteration of a Newton line search. This is approximation is satisfactory and otherwise it would be too expensive to compute $J_{u^{h}}^{h \prime \prime}$ at some other points.

3. The use of the scalar product $a^{h}(\cdot, \cdot)$ in step 1 gives a gradient $r^{h, l}$ which is again a Sobolev gradient. This acts like a preconditionning on the (nonlinear) conjugate gradient method.

4. The parallel transport in step 2, which corresponds to the Riemannian metric of $\mathcal{E}_{g}^{h}$ induced by the scalar product $a^{h}(\cdot, \cdot)$, is easy to compute because it also corresponds to the parallel transport on $S^{2}$.

\subsection{Numerical results}

We used the same boundary condition and initial value as in Section 2.3 (a test-case in given by a triplet $n(a) p)$. We first comment Figures 7 and 8 .

1. In Figure 7 we compare the speed of convergence of the Sobolev gradient, Newton, and conjugate gradient algorithm. For the conjugate gradient we have set $l_{\max }=10$ (with $N \sim 1000$ ). It is useless to have a big $l_{\max }$.

The Newton algorithm is the fastest, but the test-case is not too hard (degree $p=1$ ), since there is no refinement: we recall that in general, the Newton algorithm is unstable.

The conjugate gradient converges faster than the Sobolev gradient and with a very good accuracy (the error is stabilized at $\sim 10^{-15}$ because of the computer precision $\sim 10^{-16}$ ).

It is interesting to see that the Sobolev gradient shows 2 different rate of convergence: until iteration 40 , the rate is rather high, and after iteration 40 it is slower. At iteration 40 the error is $10^{-5}$ which is good enough for our purposes (a better precision in the computation is meaningless since we already compute an approximation!).

2. Figure 8 shows the evolution of the discrete energy $E^{h}\left(u^{h, k}\right)$ as a funtion of the iteration $k$, for the two algorithms of Sobolev gradient and conjugate gradient (with $l_{\max }=10$ ). Every refinement is represented by a dashed vertical line.

In both cases the computed energy is higher than the real energy (which can be computed explicitly because the solution is known). The interpretation is that the discrete energy (with the refining strategy) stays higher than the degree and not equal because the approximation is not harmonic. This is a global argument (integration over $B^{2, h}$ ).

Each time there is a refinement, the energy increases. The argument this time is local: the energy of a linear interpolation on a triangle is lower than the energy of the interpolation on the refined triangle. The refinement stops sooner for the conjugate gradient (less than iteration 400) than for the Sobolev gradient (more than 2000). This reflects the fact that the conjugate gradient converges faster.

The (preconditionned) conjugate gradient algorithm applied for the computation of harmonic maps has the usual advantages of this type of algorithm: it is global, stable and fast. This algorithm is really interesting in the cases where there is many refinements (solutions with strong gradient like the test-case 1(10)1). It works really well because the energy that is minimized is also the norm of the Hilbert space $P^{1, h}$ : the metric induced by the hessian of the energy $E^{h}$ is close to the Riemannian metric of the submanifold $\mathcal{E}_{g}^{h}$.

\section{Appendix 1: NeWTON ALGORITHM FOR MANIFOLdS}

The Newton algorithm is classically used to find critical points of a functionnal defined on a Banach space. The aim of this section is to generalize the Newton algorithm to manifolds, possibly of infinite dimension.

The idea is to write the Newton algorithm by using a chart of the manifold. In case of a Riemannian manifold, the chart can be chosen so that it represents well not only the differential structure, but also the Riemannian metric: the "exponential map" associated to a Riemannian manifold gives such kind of charts. In that case, the chart depends on the current point and in order to prove the convergence of the algorithm, we need to take into account the differences between two charts which are given by the transition mappings. 


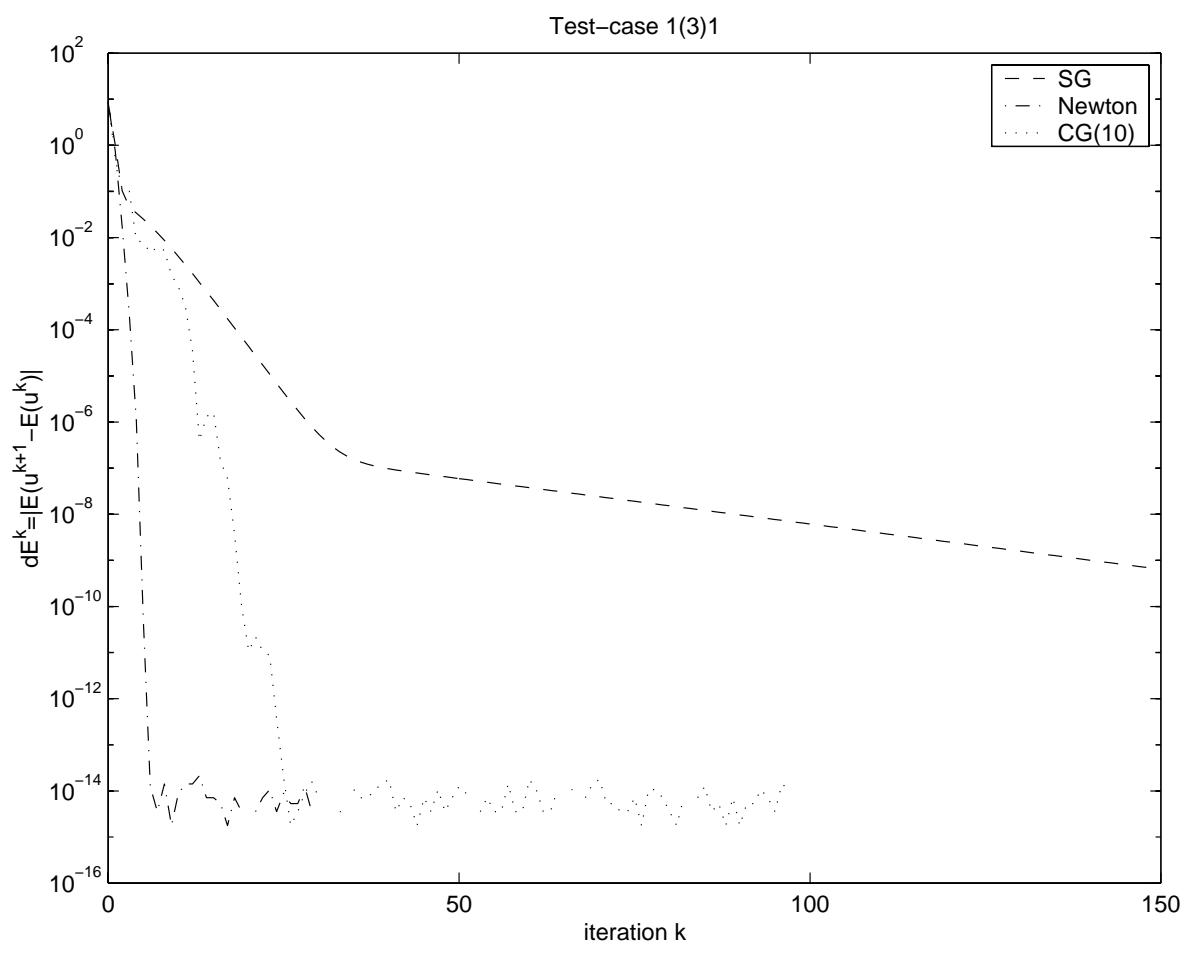

FIGURE 7. Speed of convergence (no refinement).

As in the classical Newton algorithm, we obtain a local convergence result and in particular, we prove that the convergence rate under appropriate hypotheses is quadratic.

\subsection{Newton algorithm in Banach space}

For the convenience of the reader we first recall the Newton algorithm for finding critical points (see [7,24]).

Theorem 5.1. Let $\Omega$ be an open set of a Banach space $X$ and $J: \Omega \rightarrow \mathbb{R}$ twice continuously differentiable with $J^{\prime \prime}$ locally lipschitz where $J^{\prime \prime}(x)\langle\cdot, \cdot\rangle \in \mathcal{L}\left(X, X^{\prime}\right)$ is the hessian of $J$.

Let $\underline{x} \in \Omega$ be such that $J^{\prime}(\underline{x})=0 \in X^{\prime}, J^{\prime \prime}(\underline{x}) \in \operatorname{Isom}\left(X, X^{\prime}\right)$.

Then there exists $r>0$ such that if $x^{0} \in B(\underline{x}, r)$ the sequence $\left(x^{k}\right)_{k \geq 0}$ given by $x^{k+1}=x^{k}-J^{\prime \prime}\left(x^{k}\right)^{-1} J^{\prime}\left(x^{k}\right)$ is defined for all $k \geq 0$, is contained in $B(\underline{x}, r)$ and converges to $\underline{x}$ as $k$ tends to $+\infty$. Moreover the convergence rate is quadratic, i.e. there exists $K>0$ such that $\left\|x^{k+1}-\underline{x}\right\| \leq K\left\|x^{k}-\underline{x}\right\|^{2}$.

\subsection{Hessian on a manifold}

We want to generalize the Newton algorithm to manifolds (of finite or infinite dimension).

First consider a smooth differentiable manifold $M$ modelled on the Banach space $X$, i.e. a metric separable space which is locally homeomorphic to $X$ and for which the transition mappings are smooth diffeomorphisms (see [17]). Let $J: M \rightarrow \mathbb{R}$ be a $\mathcal{C}^{2}$ function on $M$ and $\underline{m} \in M$ be a critical point of $J$, i.e. such that $T_{\underline{m}} J=0$ (where $T_{\underline{m}} J$ is the tangent mapping). Since the Newton algorithm is local we can try to find $\underline{m}$ by using a chart $(U, \varphi)$ that contains $\underline{m}$. Denoting $J_{\varphi}:=J \circ \varphi^{-1}$ defined on an open subset $\Omega$ of $X$, the Newton algorithm is:

$$
x^{k+1}=x^{k}-\left[J_{\varphi}^{\prime \prime}\left(x^{k}\right)\right]^{-1} J_{\varphi}^{\prime}\left(x^{k}\right) \quad(k \geq 0) .
$$




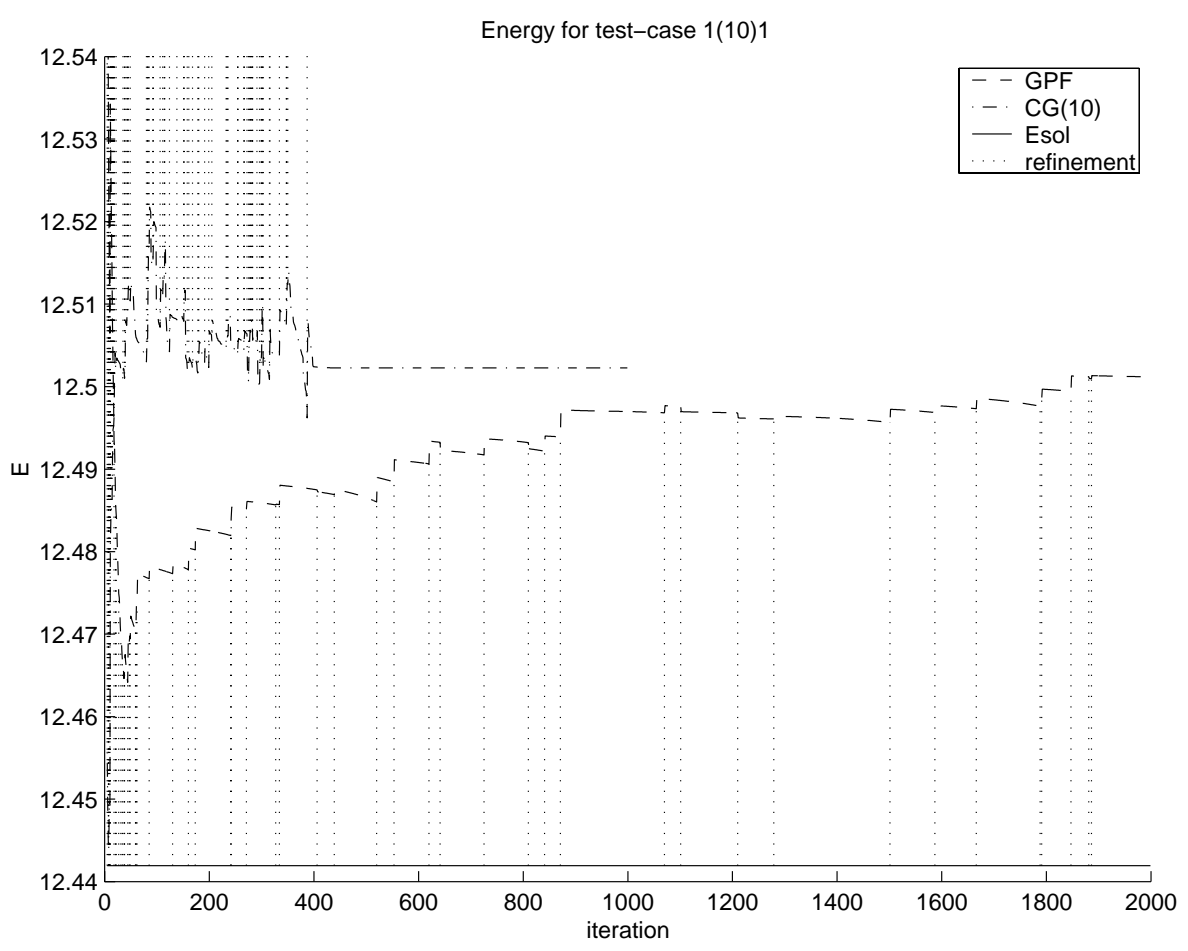

FiguRE 8. Energy and refinement $(\delta=\pi / 8)$.

The sequence $\left(m^{k}\right)_{k \geq 0}$ with $m^{k}:=\varphi^{-1}\left(x^{k}\right) \in M$ depends on the choice of the chart $(U, \varphi)$. There is indeed a notion of tangent application $T J$ independent of the chart and corresponding to $J_{\varphi}^{\prime}$, but there is no such notion as a hessian of $J$ corresponding to the hessian $J_{\varphi}^{\prime \prime}$ independtly from $\varphi$. Let $(V, \psi)$ be another chart that contains $\underline{m}$ and $J_{\psi}:=J \circ \psi^{-1}$ defined on an open subset of $X$. We want to examine the relationship between the derivatives of $J_{\varphi}$ and $J_{\psi}$. Denote $\phi:=\psi \circ \varphi^{-1}$ and $y=\phi(x)$. Then $J_{\psi}(\phi(x))=J_{\varphi}(x)$ so

$$
J_{\psi}^{\prime}(\phi(x)) \circ \phi^{\prime}(x)=J_{\varphi}^{\prime}(x)
$$

and

$$
J_{\psi}^{\prime \prime}(\phi(x))\left\langle\phi^{\prime}(x) \cdot, \phi^{\prime}(x) \cdot\right\rangle+J_{\psi}^{\prime}(\phi(x)) \circ \phi^{\prime \prime}(x)\langle\cdot, \cdot\rangle=J_{\varphi}^{\prime \prime}(x)\langle\cdot, \cdot\rangle
$$

where $J_{\psi}^{\prime}(y)$ and $J_{\varphi}^{\prime}(x)$ are in the topological dual $X^{\prime}, J_{\psi}^{\prime \prime}(y)\langle\cdot, \cdot\rangle$ and $J_{\phi}(x)\langle\cdot, \cdot\rangle$ are in $\mathcal{L}_{c}\left(X, X^{\prime}\right), \phi^{\prime}(x)$ is in $\operatorname{Isom}(X)$ and $\phi^{\prime \prime}(x)\langle\cdot, \cdot\rangle$ is in $\mathcal{L}_{c}\left(X, \mathcal{L}_{c}(X)\right)$.

Notice that $J_{\psi}^{\prime \prime}$ and $J_{\varphi}^{\prime \prime}$ are the same (up to the change of coordinates $\phi^{\prime}(x)$ ) if $J_{\varphi}^{\prime}(x)=0$ (critical point) or $\phi^{\prime \prime}(x)\langle\cdot, \cdot\rangle=0$ (affine change of coordinates).

In order to state a Newton convergence theorem for manifolds we also need some regularity of the hessian. The proposition contained in the following definition is obvious by (5.3).

Definition 5.1. Let $M$ be a smooth manifold modelled on a Banach space $X$ and $J: M \rightarrow \mathbb{R}$ a $\mathcal{C}^{2}$ functionnal on $M$. We say (with abusive notations) that $J^{\prime \prime}$ is locally Lipschitz continuous if one of the following equivalent conditions is satisfied:

1. For all $m \in M$ there exists a chart $(U, \varphi)$ with $U$ containing $m$ such that $J_{\varphi}^{\prime \prime}$ is locally Lipschitz continuous (where $J_{\varphi}:=J \circ \varphi^{-1}$ );

2. For every chart $(U, \varphi)$ of the atlas, $J_{\varphi}^{\prime \prime}$ is locally lipschitz continuous. 


\subsection{Newton algorithm for Riemannian manifold}

For our application we deal with Riemannian manifolds modelled on a Hilbert space. If we want to define an algorithm that takes into account the Riemannian structure of $M$ we need to choose a good chart, for instance the exponential application. Let $M$ be a Riemannian manifold modelled on the (separable) Hilbert space $H$ and $J: M \rightarrow \mathbb{R}$ a $\mathcal{C}^{2}$ functionnal on $M . M$ is a metric space equipped with the geodesic distance $d(\cdot, \cdot)$ and every tangent space $T_{m} M$ for $m \in M$ has the hilbertian norm $\|\cdot\|_{T_{m} M}$. Let $T M$ be the tangent bundle considered with its differential structure and $\Pi: T M \rightarrow M$ the canonical projection on $M$.

Assumptions 5.1. We suppose that for every $m \in M$ we have a mapping $\varphi_{m}: \Omega_{m} \rightarrow M$ defined on an open neighbourhood $\Omega_{m}$ of 0 in $T_{m} M$ and that satisfies the following properties:

1. $\varphi_{m}(0)=m$ and $T_{0} \varphi_{m}=I d_{T_{m} M}$;

2. $\Omega(M):=\sqcup_{m \in M} \Omega_{m}$ is an open subset of the tangent bundle $T M$ and $\varphi: v \in \Omega(M) \rightarrow \varphi_{\Pi(v)}(v) \in M$ is of class $\mathcal{C}^{\infty}$.

\section{Remarks.}

1. By the inverse mapping theorem, for every $m \in M, \varphi_{m}^{-1}$ exists and defines a chart.

2. The exponential mapping defined for a Riemannian manifold satisfies the required properties.

3. The main problem in order to implement the Algorithm 5.1 below is to find a map $\varphi_{m}$ easy to compute. In our examples of harmonic maps with values in $S^{2}$ this is possible. We actually use a mapping which is close to the exponential application but easier to compute.

Define for all $m \in M J_{m}=J \circ \varphi_{m}$. Let $\underline{m} \in M$ be such that $T_{\underline{m}} J=0$ and $J_{m}^{\prime \prime}(0) \in \operatorname{Isom}\left(T_{\underline{m}} M, T_{\underline{m}} M^{\prime}\right)$. The algorithm is given by:

Algorithm 5.1 (Newton for manifolds).

Initial Data. Set $k=0$ and choose $m^{0} \in M$.

Step 1. Compute the search direction

$$
v^{k}=-\left[J_{m^{k}}^{\prime \prime}(0)\right]^{-1} \cdot J_{m^{k}}^{\prime}(0) \in T_{m^{k}} M .
$$

Step 2. Set

$$
m^{k+1}=\varphi_{m^{k}}\left(v^{k}\right) \in M
$$

Replace $k$ by $k+1$, and go to step 1 .

Remark. If $M=E$ is a Banach space and $\varphi_{m}=I d_{E}$ then this algorithm is the classical Newton algorithm.

We can now state:

Theorem 5.2. Let $M$ be a Riemannian manifold modelled on a (separable) Hilbert space and $J: M \rightarrow \mathbb{R}$ a $\mathcal{C}^{2}$ function on $M$ with $J^{\prime \prime}$ locally Lipschitz continuous. Let $\varphi$ satisfie assumptions 5.1 and set $J_{m}=J \circ \varphi_{m}$.

Let $\underline{m} \in M$ be such that $T_{\underline{m}} J=0$ and $J_{\underline{m}}^{\prime \prime}(0) \in I \operatorname{som}\left(T_{\underline{m}} M, T_{\underline{m}} M^{\prime}\right)$.

Then there exists $r>0$ such that if $m^{\overline{0}} \in B_{\underline{m}}(r)$, the sequence $\left(m^{k}\right)_{k \geq 0}$ constructed by the Newton Algorithm 5.1 is defined for every $k \geq 0$, contained in $B_{\underline{m}}(r)$ and converges to $\underline{m}$ as $k$ tends to $+\infty$. Moreover the convergence rate is quadratic, i.e. there exists $K>0$ such that

$$
d\left(m^{k+1}, \underline{m}\right) \leq K d\left(m^{k}, \underline{m}\right)^{2} .
$$

In this theorem $d(\cdot, \cdot)$ is the geodesic distance and $B_{\underline{m}}(r)$ is the ball of center $\underline{m}$ and radius $r$.

\subsection{Proof}

5.4.1. Version in the tangent space

We start writing the Newton algorithm in $T_{\underline{m}} M$ using the chart $\varphi_{m}^{-1}$ and the Hilbert space $T_{\underline{m}} M$ as model space. 
Set $y^{k}:=\varphi_{\underline{m}}^{-1}\left(m^{k}\right)$. For $m=\varphi_{\underline{m}}(y)$ close to $\underline{m}$ (and $y \in T_{\underline{m}} M$ ) the transition mapping is $\phi_{y}:=\varphi_{\underline{m}}^{-1} \circ \varphi_{m}$ defined from an open neighbourhood of 0 in $T_{m} M$ into an open neighbourhood of 0 in $T_{\underline{m}} M$.

By definition, $y^{k+1}=\phi_{y^{k}}\left(-\left[J_{m^{k}}^{\prime \prime}(0)\right]^{-1} \cdot J_{m^{k}}^{\prime}(0)\right)$. Using (5.2), (5.3) and $y^{k}=\phi_{y^{k}}(0)$ we find

$$
J_{m^{k}}^{\prime}(0)=J_{\underline{m}}^{\prime}\left(\phi_{y^{k}}(0)\right) \circ \phi_{y^{k}}^{\prime}(0) \quad\left(0 \in T_{m^{k}} M\right),
$$

and

$$
J_{m^{k}}^{\prime \prime}(0)\langle\cdot, \cdot\rangle=J_{\underline{m}}^{\prime \prime}\left(\phi_{y^{k}}(0)\right)\left\langle\phi_{y^{k}}^{\prime}(0) \cdot, \phi_{y^{k}}^{\prime}(0) \cdot\right\rangle+J_{\underline{m}}^{\prime}\left(\phi_{y}^{k}(0)\right) \circ \phi_{y^{k}}^{\prime \prime}(0)\langle\cdot, \cdot\rangle .
$$

We recall that $J_{m^{k}}^{\prime \prime}(0)\langle\cdot, \cdot\rangle \in \mathcal{L}_{c}\left(T_{m^{k}} M, T_{m^{k}} M^{\prime}\right), J_{\underline{m}}^{\prime \prime}\left(y^{k}\right) \in \mathcal{L}_{c}\left(T_{\underline{m}} M, T_{\underline{m}} M\right), \phi_{y^{k}}^{\prime}(0) \in \mathcal{L}_{c}\left(T_{m^{k}} M, T_{m} M\right)$, $J_{\underline{m}}^{\prime}\left(y^{k}\right) \in \mathcal{L}_{c}\left(T_{\underline{m}} M, \mathbb{R}\right)$ and $\phi_{y^{k}}^{\prime \prime}(0)\langle\cdot, \cdot\rangle \in \mathcal{L}_{c}\left(T_{m^{k}} M,{\mathcal{\mathcal { L } _ { c }}}_{c}\left(T_{m^{k}} M, T_{\underline{m}} M\right)\right)$.

So we have

$$
\begin{array}{r}
y^{k+1}=\phi_{y^{k}}\left(-\left[J_{\underline{m}}^{\prime \prime}\left(y^{k}\right)\left\langle\phi_{y^{k}}^{\prime}(0) \cdot, \phi_{y^{k}}^{\prime}(0) \cdot\right\rangle+J_{\underline{m}}^{\prime}\left(y^{k}\right) \circ \phi_{y^{k}}^{\prime \prime}(0)\langle\cdot, \cdot\rangle\right]^{-1}\right. \\
\left.\cdot\left(J_{\underline{m}}^{\prime}\left(y^{k}\right) \circ \phi_{y^{k}}^{\prime}(0)\right)\right) .
\end{array}
$$

Proving the theorem for the sequence $m^{k}$ defined by the Newton algorithm is exactly the same as proving a similar theorem for a sequence $\tilde{y}^{k}$ that would be defined by (5.5). In order to do this and see in particular that every term is well defined we need to use the differential structure of the tangent bundle. Denote $(U, \psi)$ with $\psi:=\varphi_{\underline{m}}^{-1}$ our favorite chart. The corresponding chart on $T M$ maps $v \in T_{m} M$ to $\left(\psi(m), T_{m} \psi \cdot v\right) \in T_{\underline{m}} M \times T_{\underline{m}} M$ and is defined on the open subset of $T M \tilde{U}:=\sqcup_{m \in U} T_{m} M$. Using this, for $m=\varphi_{\underline{m}}(y)$ :

- $\phi_{y}: T_{m} M \rightarrow T_{\underline{m}} M$ becomes $\phi_{y} \circ T_{y} \varphi_{\underline{m}}: T_{\underline{m}} M \rightarrow T_{\underline{m}} M$;

- $\phi_{y}^{\prime}(0): T_{m} M \stackrel{\underline{m}}{\rightarrow} T_{\underline{m}} M$ becomes $\phi_{y}^{\prime}(0) \circ \frac{\underline{m}}{T_{y}} \varphi_{\underline{m}}=\underline{I} d_{T_{\underline{m}} M}$. Indeed, $\phi_{y}^{\prime}(0)=\left[T_{y} \varphi_{\underline{m}}\right]^{-1} \circ T_{0} \varphi_{m}$ with $T_{0} \varphi_{m}=I d_{T_{m} M}$

- $\phi_{y}^{\prime \prime}(0)\langle\cdot, \cdot\rangle \in \mathcal{L}_{c}\left(T_{m} M, \mathcal{L}_{c}\left(T_{m} M, T_{\underline{m}} M\right)\right)$ becomes

$$
\phi_{y}^{\prime \prime}(0)\left\langle T_{y} \varphi_{\underline{m}} \cdot T_{y} \varphi_{\underline{m}} \cdot\right\rangle \in \mathcal{L}_{c}\left(T_{\underline{m}} M, \mathcal{L}_{c}\left(T_{\underline{m}} M, T_{\underline{m}} M\right)\right) .
$$

Hence

$$
\begin{aligned}
y^{k+1}=\left(\phi_{y^{k}}\right. & \left.\circ T_{y^{k}} \varphi_{\underline{m}}\right)\left(-\left[J_{\underline{m}}^{\prime \prime}\left(y^{k}\right)\langle\cdot, \cdot\rangle\right.\right. \\
& \left.\left.+J_{\underline{m}}^{\prime}\left(y^{k}\right) \circ \phi_{y^{k}}^{\prime \prime}(0)\left\langle T_{y^{k}} \varphi_{\underline{m}}, T_{y^{k}} \varphi_{\underline{m}} \cdot\right\rangle\right]^{-1} \cdot J_{\underline{m}}^{\prime}\left(y^{k}\right)\right) .
\end{aligned}
$$

We are therefore led to prove the following lemma:

Lemma 5.1. There exists $r>0$ such that if $y^{0} \in B(0, r)$, the sequence $\left(y^{k}\right)_{k>0}$ given by (5.6) is defined for all $k \geq 0$, contained in $B(0, r)$ and converges to 0 as $k$ tends to $\infty$. Moreover the convergence rate is quadratic, i.e. there exists $K>0$ such that

$$
\left\|y^{k+1}\right\|_{T_{\underline{m}} M} \leq K\left\|y^{k}\right\|_{T_{\underline{m}} M}^{2} .
$$

Suppose indeed that Lemma 5.1 is proved.

If $m^{0}$ is close enough to $\underline{m}$, then $y^{0}:=\varphi_{\underline{m}}^{-1}\left(m^{0}\right)$ is close enough to 0 and the sequence $\left(y^{k}\right)$ is well defined and converges to 0 . Thus the sequence $\left(m^{\prime k} \bar{k}\right)$ with $m^{\prime k}:=\varphi_{m}\left(y^{k}\right)$ is well defined and converges to $\underline{m}$. By the computation above, this sequence is exactly the one defined by the Newton Algorithm 5.1. We only need to prove that the convergence rate is quadratic (in the sense of (5.4) for the sequence $\left(m^{\prime k}\right)=\left(m^{k}\right)$.

Suppose first that $\varphi_{\underline{m}}=\exp _{\underline{m}}$. Then by property of the exponential mapping $\|y\|_{T_{\underline{m}} M}=d\left(\exp _{\underline{m}}(y), \underline{m}\right)$ (for all $y \in T_{\underline{m}} M$ small enough), and Theorem 5.2 is proved. 
In the general case, $d\left(\varphi_{\underline{m}}(y), \underline{m}\right)=\left\|\exp _{\underline{m}}^{-1} \circ \varphi_{\underline{m}}(y)\right\|_{T_{\underline{m}} M}$ and since $\exp _{\underline{m}}^{-1} \circ \varphi_{\underline{m}}(y)=y+O\left(\|y\|^{2}\right)$ by assumptions 5.1 on $\varphi$ and property of the exponential mapping, there exists $C_{1}$ and $\bar{C}_{2}$ with $0<C_{1} \leq 1 \leq C_{2}$ such that

$$
C_{1}\|y\|_{T_{\underline{m}} M} \leq d\left(\varphi_{\underline{m}}(y), \underline{m}\right) \leq C_{2}\|y\|_{T_{\underline{m}} M} .
$$

Set $r^{\prime}=\min \left\{C_{1} r, \frac{C_{1}}{K C_{2}}\right\}$. Then if $m^{k} \in B_{\underline{m}}\left(r^{\prime}\right),\left\|y^{k}\right\| \leq \min \left\{r, \frac{1}{K C_{2}}\right\}$, and $K\left\|y^{k}\right\| \leq C_{1} / C_{2}$ so by (5.6) $\left\|y^{k+1}\right\| \leq \frac{r^{\prime}}{C_{2}}$ and $m^{k+1} \in B_{\underline{m}}\left(r^{\prime}\right)$. Therefore if $m^{0} \in B_{\underline{m}}\left(r^{\prime}\right)$, Theorem 5.2 is proved with $d\left(m^{k+1}, \underline{m}\right) \leq$ $\frac{K C_{2}}{C_{1}^{2}} d\left(m^{k}, \underline{m}\right)$.

\subsubsection{Proof of Lemma 5.1.}

Define $G_{y}(v):=\phi_{y} \circ T_{y} \varphi_{\underline{m}} \cdot v$ and

$$
F(y):=-\left[J_{\underline{m}}^{\prime \prime}(y)\langle\cdot, \cdot\rangle+J_{\underline{m}}^{\prime}(y) \circ \phi_{y}^{\prime \prime}(0)\left\langle T_{y} \varphi_{\underline{m}} \cdot T_{y} \varphi_{\underline{m}} \cdot\right\rangle\right]^{-1} \cdot J_{\underline{m}}^{\prime}(y) \in T_{\underline{m}} M
$$

By regularity of $\varphi$, the mapping $K:(y, v) \rightarrow \varphi_{\varphi_{\underline{m}}(y)} \circ T_{y} \varphi_{\underline{m}} \cdot v$ is defined and of class $\mathcal{C}^{\infty}$ on $B\left(0, r_{1}\right) \times B\left(0, r_{2}\right)$ (as an open subset of $T_{\underline{m}} M \times T_{\underline{m}} M$ ) for $r_{1}>0, r_{2}>0$ small enough. So,

$$
\begin{aligned}
& (y, v) \rightarrow G_{y}(v)=\varphi_{\underline{m}}^{-1}(K(y, v))=\phi_{y} \circ T_{y} \varphi_{\underline{m}} \cdot v \text { is of class } \mathcal{C}^{\infty} \text { on } B\left(0, r_{1}\right) \times B\left(0, r_{2}\right) ; \\
& y \rightarrow \frac{\partial G}{\partial v}(y, 0)=\phi_{y}^{\prime}(0) \circ T_{y} \varphi_{\underline{m}} \cdot \text { and } y \rightarrow \frac{\partial^{2} G}{\partial v^{2}}(y, 0)\langle\cdot, \cdot\rangle=\phi_{y}^{\prime \prime}(0)\left\langle T_{y} \varphi_{\underline{m}}, T_{y} \varphi_{\underline{m}} \cdot\right\rangle \text { is defined and of class } \\
& \mathcal{C}^{\infty} \text { on } B\left(0, r_{2}\right) .
\end{aligned}
$$

By regularity of $J_{\underline{m}}$ there exists $r_{3}>0$ such that $J_{\underline{m}}$ is of class $\mathcal{C}^{2}$ on $B\left(0, r_{3}\right)$; the mapping $H: y \rightarrow J_{\underline{m}}^{\prime \prime}(y)\langle\cdot, \cdot\rangle+$ $J_{\underline{m}}^{\prime}(y) \circ \phi_{y}^{\prime \prime}(0)\left\langle T_{y} \varphi_{\underline{m}} \cdot, T_{y} \varphi_{\underline{m}} \cdot\right\rangle$ is therefore continuous on $B\left(0, r_{4}\right)$ (with $\left.r_{4}:=\min \left\{r_{2}, r_{3}\right\}\right)$. Recall that $J_{\underline{m}}^{\prime}(0)=0$ so $H(0)=J_{\underline{m}}^{\prime \prime}(0) \in I \operatorname{som}\left(T_{\underline{m}} M, T_{\underline{m}} M^{\prime}\right)$; by continuity and the resolvant formula (see [4]) there exists $\left.r_{5} \in\right] 0, r_{4}$ ] such that $\overline{H(}(y) \in \operatorname{Isom}\left(T_{\underline{m}} M, T_{\underline{m}} M^{\prime}\right)$ for all $y \in B\left(0, r_{5}\right)$ and $y \rightarrow[H(y)]^{-1}$ is bounded on $B\left(0, r_{5}\right)$. Set $M:=\sup _{y \in B\left(0, r_{5}\right)}\left\|H(y)^{-1}\right\| ;$ by continuity there exists $0<r_{6}<r_{3}$ such that $\left\|J_{\underline{m}}^{\prime}(y)\right\| \leq r_{2} / M$ for all $y \in B\left(0, r_{6}\right)$. Set at last $r_{7}:=\min \left\{r_{5}, r_{6}, r_{1}, r_{2}\right\}$; then for all $y \in B\left(0, r_{7}\right), F(y)$ is defined with $\|F(y)\| \leq r_{2}$ and $G(y, F(y))$ is defined.

In order to conclude the proof we need two lemmas.

Lemma 5.2. There exists $\left.\left.r_{8} \in\right] 0, r_{7}\right]$ and $K>0$ such that $\|F(y)+y\| \leq K\|y\|^{2}$ for all $y \in B\left(0, r_{8}\right)$.

Lemma 5.3. There exists $\left.\left.r_{9} \in\right] 0, r_{8}\right]$ and $K^{\prime}>0$ such that $\|G(y, F(y))\| \leq K^{\prime}\|y\|^{2}$ for all $y \in B\left(0, r_{9}\right)$.

Postponing the proof of Lemmas 5.2 and 5.3, choose $\alpha \in] 0,1\left[\right.$ and set $r=\min \left\{r_{9}, \alpha / K^{\prime}\right\}$. Then if $y^{k} \in B(0, r)$, by Lemma $5.3 y^{k+1}:=G\left(y^{k}, F\left(y^{k}\right)\right)$ satisfies

$$
\left\|y^{k+1}\right\| \leq K^{\prime}\left\|y^{k}\right\|^{2}
$$

So

$$
\left\|y^{k+1}\right\| \leq \alpha\left\|y^{k}\right\|
$$

and in particular $y^{k+1} \in B(0, r)$. If we choose $y^{0} \in B(0, r)$, by induction the entire sequence is defined and contained in $B(0, r)$, and by (5.9) converges to 0 ; by (5.8) the convergence rate is quadratic and the proof is complete. 
Proof of Lemma 5.2. For $y \in B\left(0, r_{7}\right)$,

$$
\begin{aligned}
F(y)+y= & -[H(y)\langle\cdot, \cdot\rangle]^{-1} \cdot J_{\underline{m}}^{\prime}(y)+[H(y)\langle\cdot, \cdot\rangle]^{-1} \cdot H(y)\langle y, \cdot\rangle, \\
= & {[H(y)]^{-1}\left(-\int_{0}^{1} J_{\underline{m}}^{\prime \prime}(s y)\langle y, \cdot\rangle \mathrm{d} s+J_{\underline{m}}^{\prime \prime}(y)\langle y, \cdot\rangle\right.} \\
= & \left.\left.\quad+J_{m}^{\prime}(y) \circ \phi_{y}^{\prime \prime}(0)<T_{y} \varphi_{\underline{m}} y, T_{y} \varphi_{\underline{m}} \cdot\right\rangle\right) \mathrm{d} s \\
= & {[H(y)\langle\cdot, \cdot\rangle]^{-1}\left(\int_{0}^{1}\left[J_{\underline{m}}^{\prime \prime}(y)-J_{\underline{m}}^{\prime \prime}(s y)\right]\langle y, \cdot\rangle \mathrm{d} s\right.} \\
& \left.+J_{\underline{m}}^{\prime}(y) \circ \underline{\phi}_{y}^{\prime \prime}(0)\left\langle T_{y} \varphi_{\underline{m}} y, T_{y} \varphi_{\underline{m}} \cdot\right\rangle\right) .
\end{aligned}
$$

Using that $J_{\underline{m}}^{\prime \prime}$ is a locally lipschitz mapping there exists $\left.\left.r_{7}^{\prime} \in\right] 0, r_{7}\right]$ and $K_{1}>0$ such that

$$
\left\|J_{\underline{m}}^{\prime \prime}(y)-J_{\underline{m}}^{\prime \prime}(s y)\right\| \leq K_{1}(1-s)\|y\| \quad\left(\forall s \in[0,1], \quad \forall y \in B\left(0, r_{7}^{\prime}\right)\right) .
$$

Since $J_{\underline{m}}^{\prime}$ is of class $\mathcal{C}^{1}$ with $J_{\underline{m}}^{\prime}(0)=0$ and by continuity of $\phi_{y}^{\prime \prime}(0)$ there exist $\left.r_{8} \in\right] 0, r_{7}^{\prime}\left[, K_{2}>0\right.$ and $M_{1}>0$ such that $\left\|J_{\underline{m}}^{\prime}(y)\right\| \leq K_{2}\|y\|$ and $\left\|\phi_{y}^{\prime \prime}(0)\left\langle T_{y} \varphi_{\underline{m}}, T_{y} \varphi_{\underline{m}} \cdot\right\rangle\right\| \leq M_{1}$ for all $y \in B\left(0, r_{8}\right)$; thus for all $y \in B\left(0, r_{8}\right)$

$$
\|F(y)+y\| \leq M\left(\frac{K_{1}}{2}+K_{2} M_{1}\right)\|y\|^{2},
$$

where $M$ is defined above. Choosing $K=M\left(\frac{K_{1}}{2}+K_{2} M_{1}\right)$ the proof is complete.

Proof of Lemma 5.3. First, recalling $G(y, v)=\varphi_{\underline{m}}^{-1} \circ \varphi_{\varphi_{\underline{m}}(y)} \circ T_{y} \varphi_{\underline{m}} \cdot v$ and assumptions on $\varphi_{m}$ we easily compute the derivatives of $G$ :

$$
\frac{\partial G}{\partial y}(0,0)=I d_{T_{\underline{m}} M} \quad \text { and } \quad \frac{\partial G}{\partial v}(0,0)=I d_{T_{\underline{m}} M} .
$$

Then using $G(0, F(0))=0$ we write:

$$
G(y, F(y))=[G(y, F(y))-G(0, F(y))-y]+[G(0, F(y))-G(0, F(0))+y] .
$$

Denote $S_{1}(y)\left(S_{2}(y)\right.$, respectively) the first (second, resp.) term into brackets in (5.11). Then for $y \in B\left(0, r_{7}\right)$,

$$
S_{1}(y)=\int_{0}^{1}\left[\frac{\partial G}{\partial y}(s y, F(y)) \cdot y-\frac{\partial G}{\partial y}(0,0) \cdot y\right] \mathrm{d} s .
$$

Since $\frac{\partial G}{\partial y}$ is $\mathcal{C}^{\infty}$ and $F(y)$ is small by Lemma 5.2 , there exist $\left.\left.r_{8}^{\prime} \in\right] 0, r_{8}\right]$ and $K_{2}$ such that

$$
\left\|\frac{\partial G}{\partial y}(s y, F(y))-\frac{\partial G}{\partial y}(0,0)\right\| \leq K_{2}(s\|y\|+\|F(y)\|) \quad\left(\forall s \in[0,1], \quad \forall y \in B\left(0, r_{8}^{\prime}\right)\right) .
$$

Using again Lemma 5.2 we find $\|F(y)\| \leq C\|y\|$ for all $y \in B\left(0, r_{8}\right)$ with $C=1+K r_{8}$; so

$$
\left\|S_{1}(y)\right\| \leq(1+C)\|y\|^{2} \quad\left(\forall y \in B\left(0, r_{8}^{\prime}\right)\right) .
$$

In a similar way,

$$
\begin{aligned}
S_{2}(y) & =\int_{0}^{1} \frac{\partial G}{\partial v}(0, s F(y)) \cdot F(y) \mathrm{d} s+\frac{\partial G}{\partial v}(0,0) \cdot y, \\
& =\left(\int_{0}^{1}\left[\frac{\partial G}{\partial v}(0, s F(y))-\frac{\partial G}{\partial v}(0,0)\right] \cdot F(y) \mathrm{d} s\right)+(F(y)+y) .
\end{aligned}
$$


There exist $\left.\left.r_{9} \in\right] 0, r_{8}^{\prime}\right]$ and $K_{3}$ such that

$$
\left\|\frac{\partial G}{\partial v}(0, s F(y))-\frac{\partial G}{\partial v}(0,0)\right\| \leq K_{3} s\|F(y)\| \quad\left(\forall s \in[0,1] \forall y \in B\left(0, r_{9}\right)\right),
$$

so using Lemma 5.2,

where $C$ is defined above.

$$
\left\|S_{2}(y)\right\| \leq \frac{K_{3} C^{2}}{2}\|y\|^{2}+K\|y\|^{2} \quad\left(\forall y \in B\left(0, r_{9}\right)\right)
$$

Choosing $K^{\prime}=1+C+\frac{K_{3} C^{2}}{2}+K$ we get $\|G(y, F(y))\| \leq K^{\prime}\|y\|^{2}$ for all $y \in B\left(0, r_{9}\right)$ and the proof is complete.

\section{Appendix 2: CONJUGATE GRAdient FOR RIEMANNiAn MANifolds}

The Newton algorithm converges to critical points of a functional and might not converge globally. Since we are interested in minima, a global minimisation algorithm would be better: the (nonlinear) conjugate gradient $[7,9,24,29]$ is such an algorithm, but it is known in Hilbert spaces. In this section, in the same way as we generalized the Newton algorithm, we generalize the nonlinear conjugate gradient algorithm (NLCG) to Riemannian manifolds.

The idea is to write the NLCG in a local chart defined by the exponential mapping associated to the Riemannian manifold. One problem is that the chart depends on the current point $m^{k}$, and in order to compute the descent direction $d^{k}$ in the tangent space $T_{m^{k}}$ at iteration $k$, we need the descente direction $d^{k-1}$ from the previous iteration $k-1$. Yet $d^{k-1}$ belongs to the tangent space $T_{m^{k-1}}$ : so we use a parallel transport to move $d^{k-1}$ from $T_{m^{k-1}}$ to $T_{m^{k}}$.

\subsection{NLCG in Hilbert space}

We first recall the NLCG algorithm [7,24,29]:

Algorithm 6.1 (NLCG). Let $H$ be a (separable) Hilbert space of dimension dim $_{H}$ (possibly infinite) with scalar product $\langle\cdot, \cdot\rangle$ and norm $\|\cdot\|$. Let $\Omega$ be an open subset of $H$ and $J: \Omega \rightarrow \mathbb{R}$ twice continuously differentiable. Let $\underline{x} \in \Omega$ be a local minimum for $J$ such that $J^{\prime \prime}(\underline{x})$ is a continuous coercive symmetric bilinear form on $H$. The NLCG for finding $\underline{x}$ is:

\section{Initial Data.}

- Set $k=0$ and choose a starting point $x^{k}$.

- Choose an integer $l_{\max } \leq \operatorname{dim}_{H}$ and set $l=0$.

Step 1. Find $r^{l} \in H$ such that

$$
<r^{l}, w>=J^{\prime}\left(x^{k}\right) \cdot w \quad \forall w \in H
$$

Step 2. If $l=0$ set $d^{l}=r^{l}$, else set $d^{l}=r^{l}+\beta_{F R} d^{l-1}$ where $\beta_{F R}=\frac{\left\|r^{l}\right\|^{2}}{\left\|r^{l-1}\right\|^{2}}$.

Step 3. If $<r^{l}, d^{l}>\leq 0$ set $l=0$ and $d^{l}=r^{l}$.

Step 4. Find $\mu^{l} \in \mathbb{R}$ such that $J\left(x^{k}+\mu^{l} d^{l}\right)=\inf _{\mu \in \mathbb{R}} J\left(x^{k}+\mu d^{l}\right)$.

Step 5.

- Set $x^{k+1}=x^{k}+\mu^{l} d^{l}$.

- Replace $l$ by $l+1$ and $k$ by $k+1$.

- If $l=l_{\max }$ set $l=0$.

- Go to step 1 (until convergence).

\section{Remarks.}

1. The vector $r^{l}$ in step $\mathbf{1}$ is the gradient of $J$. The existence of $r^{l}$ is given by the Riesz representation theorem. 
2. In step 2, $\beta_{F R}$ is the choice of Fletcher-Reeves [9]. Another usual choice is $\beta_{P R}=\frac{<r^{l}, r^{l}-r^{l-1}>}{\left\|r^{l-1}\right\|^{2}}$ (Polak-Ribière [24]).

3. There are two counting indices $k$ and $l$ because we reinitialize the direction every $l_{\max }$ step. This is the meaning of the third point in step 5 .

4. If $J$ is a quadratic functional this is the usual CG algorithm, and $\beta_{F R}=\beta_{P R}$. In this case there is no step 3, there is only one counting indices $l=k$ and the algorithm converges in at most $\operatorname{dim}_{H}$ steps.

5. In step 3, we check if $d^{k}$ is a good descent vector. If it is too bad we use the gradient as descent direction and restart the algorithm.

6. Step 4 is usually solved by a Newton line search.

\subsection{Conjugate gradient for Riemannian manifolds}

We use the notations of Section 5.1. $M$ is a smooth Riemanian manifold modelled on the separable Hilbert space $H .\left(\varphi_{m}\right)_{m \in M}$ is a collection of mappings satisfying Assumption 5.1. $J: M \rightarrow \mathbb{R}$ is a functionnal twice continuously differentiable on $M$ and $\underline{m} \in M$ a local minimum for $J$.

The idea for finding $\underline{m}$ is to write the NLCG algorithm using the charts $\varphi_{m}$. Since the chart used changes at every step of the algorithm, we need to know what the old direction $d^{k-1}$ used in step 2 looks like in the new chart. We solve this difficulty by parallel transport of $d^{k-1}$.

Define for all $m \in M J_{m}=J \circ \varphi_{m}$. Suppose $\underline{m}$ is such that $J_{\underline{m}}^{\prime \prime}(0)\langle\cdot, \cdot\rangle$ is a continuous coercive symmetric bilinear form. Let $\operatorname{dim}_{M}$ be the dimension of $M$ (possibly infinite). Recall that for every $m \in M$ the tangent space $T_{m} M$ is equipped with the scalar product $\langle\cdot, \cdot\rangle_{m}$; we denote $\|\cdot\|_{m}$ the norm in $T_{m} M$.

We obtain the following algorithm:

Algorithm 6.2 (Conjugate gradient for Riemannian manifolds).

Initial Data.

- Set $k=0$ and choose a starting point $m^{k} \in M$.

- Choose an integer $l_{\max } \leq \operatorname{dim}_{H}$ and set $l=0$.

Step 1. Find $r^{l} \in T_{m^{k}} M$ such that

$$
\left\langle r^{l}, w\right\rangle_{m^{k}}=J_{m^{k}}^{\prime}(0) \cdot w \quad \forall w \in T_{m^{k}} M
$$

Step 2. If $l \geq 1$ compute $\bar{d}^{l-1} \in T_{m^{k}} M$ by parallel transport of $d^{l-1}$ from $T_{m^{k-1}} M$ to $T_{m^{k}} M$.

Step 3. If $k=0$ set $d^{l}=r^{l}$, else set $d^{l}=r^{l}+\beta_{F R} \bar{d}^{-1}$ where

$$
\beta=\frac{\left\|r^{l}\right\|_{m^{k}}^{2}}{\left\|r^{l-1}\right\|_{m^{k-1}}^{2}}
$$

Step 4. If $\left\langle r^{l}, d^{l}\right\rangle_{m^{k}} \leq 0$ set $l=0$ and $d^{l}=r^{l}$.

Step 5. Find $\mu^{l} \in \mathbb{R}$ such that $J_{m^{k}}\left(\mu^{l} d^{l}\right)=\inf _{\mu \in \mathbb{R}} J_{m^{k}}\left(\mu d^{l}\right)$.

Step 6.

- Set $m^{k+1}=\varphi_{m^{k}}\left(\mu^{l} d^{l}\right)$.

- Replace $l$ by $l+1$ and $k$ by $k+1$.

- If $l=l_{\max }$ set $l=0$.

- Go to step 1 (until convergence).

\section{REFERENCES}

[1] F. Alouges, A new algorithm for computing liquid crystal stable configurations: the harmonic mapping case. SIAM J. Numer. Anal. 34 (1997) 1708-1726.

[2] F. Alouges and B.D. Coleman, Numerical bifurcation of equilibria of nematic crystals between non-co-axial cylinders. Math. Models Methods Appl. Sci. 11 (2001) 459-473. 
[3] D. Braess, Finite elements, in Theory, fast solvers, and applications in solid mechanics. Translated from the 1992 German edition by Larry L. Schumaker. Cambridge University Press, Cambridge, 2nd edn. (2001).

[4] H. Brézis, Analyse fonctionnelle. Masson (1996).

[5] H. Brézis and J.-M. Coron, Large solutions for harmonic maps in two dimensions. Comm. Math. Phys. 92 (1983) 203-215.

[6] K.-C. Chang, W.-Y. Ding and R. Ye, Finite-time blow up of the heat flow of harmonic maps from surfaces. J. Differ. Geom. 36 (1992) 507-515.

[7] P.G. Ciarlet, Introduction à l'analyse numérique matricielle et à l'optimisation. Masson (1988).

[8] P.-G. De Gennes and J. Prost, The physics of liquid crystals. Clarendon Press, Oxford (1993).

[9] R. Fletcher and C.M. Reeves, Function minimization by conjugate gradients. Comput. J. 7 (1994) $149-154$.

[10] M. Giaquinta, G. Modica and J. Souček, Cartesian currents in the calculus of variations. I. Springer-Verlag, Berlin (1998).

[11] M. Giaquinta, G. Modica and J. Souček, Cartesian currents in the calculus of variations. II. Springer-Verlag, Berlin (1998).

[12] R.M. Hardt, Singularities of harmonic maps. Bull. Amer. Math. Soc. (N.S.) 34 (1997) 15-34.

[13] E. Hebey, Introduction à l'analyse non linéaire sur les variétés. Diderot Editeur Arts et Sciences (1987).

[14] F. Hélein, Régularité des applications faiblement harmoniques entre une surface et une sphère. C. R. Acad. Sci. Paris Sér. I Math. 311 (1990) 519-524.

[15] F. Hélein, Symétries dans les problèmes variationnels et applications harmoniques. Istituti Editoriali e Poligrafici Internazionali, Pisa-Roma (1998).

[16] J. Jost, Harmonic mappings betwenn surfaces. Springer-verlag, Lecture Notes in Math. 1062 (1984).

[17] W.P.A Klingenberg, Riemannian Geometry. Walter de Gruyter (1995).

[18] E. Kuwert, Minimizing the energy of maps from a surface into a 2-sphere with prescribed degree and boundary values. Manuscripta Math. 83 (1994) 31-38.

[19] L. Lemaire, Applications harmoniques de surfaces riemanniennes. J. Differ. Geom. 13 (1978) 51-78.

[20] A. Lichnewsky, Une méthode de gradient conjugué sur des variétés : application à certains problèmes de valeurs propres non linéaires. Numer. Funct. Anal. Optim. 1 (1979) 515-560.

[21] P.L. Lions, The concentration-compactness principle in the calculus of variations. The limit case, part 2. Rev. Mat. Iberoamericana 1 (1985) 45-121.

[22] C.B. Morrey, Multiple integrals in the calculus of variations. Springer, New York (1966).

[23] J.W. Neuberger, Sobolev gradients and boundary conditions for partial differential equations, in Recent developments in optimization theory and nonlinear analysis (Jerusalem, 1995), Amer. Math. Soc., Providence, RI. Contemp. Math. 204 (1997) 171-181

[24] E. Polak, Optimization, Appl. Math. Sci. 124 (1997).

[25] J. Qing, Remark on the Dirichlet problem for harmonic maps from the disc into the 2-sphere. Proc. R. Soc. Edinb. 122A (1992) 63-67.

[26] J. Qing, Boundary regularity of weakly harmonic maps from surfaces. J. Funct. Anal. 114 (1993) 63-67.

[27] R. Schoen and K. Uhlenbeck, Boundary regularity and the Dirichlet problem for harmonic maps. J. Dif. Geom. 18 (1983) $253-268$.

[28] J.R. Shewchuk, Triangle: engineering a 2d quality mesh generator and delaunay triangulator. http://www-2.cs.cmu.edu/ quake/triangle.html.

[29] J.R. Shewchuk, An introduction to the conjugate gradient method without the agonizing pain. http://www-2.cs.cmu.edu/ jrs/jrspapers.html\#cg (1994).

[30] A. Soyeur, The Dirichlet problem for harmonic maps from the disc into the 2-sphere. Proc. R. Soc. Edinb. 113A (1989) $229-234$. 